• 研究报告・

\title{
中国菌物名录数据库在大型真菌红色 名录编制中的作用
}

\author{
王 科 ${ }^{1,2}$ 赵明君 ${ }^{1}$ 苏锦河 $^{3}$ 杨 柳 ${ }^{1}$ 邓 红 ${ }^{1}$ 王永会 ${ }^{1,2}$ 吴海军 $^{1}$ \\ 李 熠 ${ }^{4}$ 吴红梅 $^{1}$ 卫晓丹 ${ }^{1}$ 魏铁铮 $^{1}$ 蔡 䂞 ${ }^{1}$ 姚一建 $^{1^{*}}$ \\ 1 (中国科学院微生物研究所真菌学国家重点实验室, 北京 100101) \\ 2 (中国科学院大学, 北京 100049) \\ 3 (集美大学计算机工程学院, 福建厦门 361021) \\ 4 (扬州大学食品科学与工程学院, 江苏扬州 225127)
}

摘要: 长期以来, 分类学的研究成果主要以平面的方式发表在各种纸质文献资料中。近年来, 随着计算机和人工 智能等新兴技术的发展和应用, 平面资料信息的数字化成为一种趋势, 世界各国都非常重视本国生物资源信息的 收集汇总, 构建了多种数据库, 为科学研究、政府决策、资源保护、合理利用和科学传播提供了重要的信息基础。 本研究探索并建立从菌物学平面资料信息构建数据库的流程和方法, 并在中国菌物名录数据库和Index Fungorum 所收集数据的基础上进行数据挖掘和分析。通过软件操作和程序设计, 在数据库中提取相关信息, 辅助完成了《中 国生物多样性红色名录一大型真菌卷》的编制工作, 同时梳理和规范了菌物拉丁和汉语学名, 为菌物分类研究 和资源评估与保护提供基础。

关键词: 文献数字化; 菌物数据库; 红色名录初评; 菌物汉语学名

\section{The use of Checklist of Fungi in China database in the red list assessment of macrofungi in China}

Ke Wang ${ }^{1,2}$, Mingjun Zhao ${ }^{1}$, Jinhe $\mathrm{Su}^{3}$, Liu Yang ${ }^{1}$, Hong Deng ${ }^{1}$, Yonghui Wang ${ }^{1,2}$, Haijun $\mathrm{Wu}^{1}$, Yi $\mathrm{Li}^{4}$, Hongmei Wu ${ }^{1}$, Xiaodan $\mathrm{Wei}^{1}$, Tiezheng $\mathrm{Wei}^{1}$, Lei Cai ${ }^{1}$, Yijian Yao ${ }^{1 *}$

1 State Key Laboratory of Mycology, Institute of Microbiology, Chinese Academy of Sciences, Beijing 100101

2 University of Chinese Academy of Sciences, Beijing 100049

3 Computer Engineering College, Jimei University, Xiamen, Fujian 361021

4 School of Food Science and Engineering, Yangzhou University, Yangzhou, Jiangsu 225127

Abstract: Taxonomic research has, to date, mainly been published in peer-based journals and books. Recently, with the development of emerging technology, document digitization has become a new trend and a variety of databases have been established to provide useful information for scientific research, government decisions, resource protection and utilization, and science communication. During the Red List Assessment of Macrofungi in China project, The Checklist of Fungi in China and Index Fungorum were used for data mining and programming methods to compile the Red List of China's Biodiversity-Macrofungi. Both Latin and Chinese scientific names of assessed species were revised and corrected, providing a useful example for established database importance in taxonomic research, evaluation, and biological conservation.

Key words: digitization; fungal databases; initial assessment; fungal Chinese name

菌物是自然界中物种多样性最为丰富的生物 类群之一，据估计，世界上分布的菌物物种可达
220-380万种(Hawksworth \& Lucking, 2017)。早在18 世纪初欧洲国家就开始了菌物分类学研究, 迄今为

收稿日期: 2019-05-13; 接受日期: 2019-07-09

基金项目: 生态环境部生物多样性调查评估项目(2019HJ2096001006)和中国科学院科技促进发展局特别支持项目

* 通讯作者 Author for correspondence. E-mail: yaoyj@im.ac.cn 
止世界范围内已发现超过 14 万个菌物物种(Species Fungorum, http://www.speciesfungorum.org, 数据获 取于2019年6月20日), 发表了大量菌物学专著和文 献, 如Systema Mycologicum (Fries, 1823)和Sylloge Fungorum: Omnium Hucusque Cognitorum (Saccardo, 1898)等。中国菌物学研究起始于 20 世纪初, 其后近 百年的发展过程中积累了如《中国的真菌》(邓叔群, 1963)、《中国真菌总汇》(戴芳澜，1979)、《真菌名 词及名称》 (中国科学院微生物研究所, 1986)和《狍 子植物名词及名称》(郑儒永等, 1990)等一系列著作, 为菌物分类学的发展积攒了丰富的资料。

随着计算机与信息技术的高速发展, 互联网以 其搜索快捷、信息量大、更新速度快等特点逐步成 为人们获取信息的主要渠道。基于网络传播的数字化 文献已逐渐成为新的趋势, 也为科学研究工作提供 了便利。在菌物方面, 世界各国已不同程度地开展了 文献数字化工作并建立了与菌物分类学相关的物种 名录数据库、分类学名称数据库、生物多样性数据 库等, 如Index Fungorum (http://www.indexfungorum. org)、MycoBank (http://www.mycobank.org)、Bibliography of Systematic Mycology (http://www.speciesf ungorum.org/BSM/bsm.htm) 等。中国的菌物文献数 字化工作起步较晚, 相关的数据库数量较少或仍在 建设完善之中。2011年, 在中国科学院生物多样性 委员会支持下，中国菌物名录数据库(http://www. fungalinfo.net)在中国科学院微生物研究所建立。该 数据库是对文献报道的中国分布的菌物种类及其 相关信息进行全面汇总和系统整理的数据集合, 包 括中国已知菌物各物种的学名、同物异名、分类地 位、国内外分布、参考文献、生境、宿主或寄主、
标本或培养物信息等。此外, 该数据库的菌物名称 注册系统Fungal Names (http://www.fungalinfo.net/ fungalname/) 已被国际菌物命名委员会授权为官方 接受的菌物名称注册网站, 且受到《国际藻类、菌 物和植物命名法规》认可(McNeil \& Turland, 2011; Turland et al, 2018)。中国菌物名录数据库的建立, 为 我国菌物系统学、生物多样性及相关领域的学术研 究、产业发展、物种保护和资源可持续利用构建了 最为全面的信息平台。

2016年，原环境保护部联合中国科学院启动了 《中国生物多样性红色名录一大型真菌卷》的编 制工作，红色名录的评估在很大程度上依赖于对某 一特定的地理或行政区域内特定物种发生的数量 和变化趋势的掌握。中国菌物名录数据库所收录的 内容为本次评估工作提供了详实的基础数据，本文 将详细介绍该数据库在此次大型真菌红色名录评 估过程中所发挥的作用, 为数据库在科学研究过程 中的使用提供实例。

\section{1 文献数字化和数据库扩充}

中国菌物名录数据库中收录的数据主要来自 于数字化的文献和书籍。在早期工作中大多依靠人 工进行信息录入，为了解决人工录入效率低、错误 率高等问题, 本研究构建了菌物平面文献数字化的 流程和方法，以进一步补充此次红色名录评估的基 础数据(图1)。

对于所收集的纸质版文献与书籍，利用扫描技 术将其逐页转化为图像并存储在电子文献库中。相 较于手工录入、语音识别等其他数字化方法，扫描 技术不仅速度快、费用低，还尽可能地保持了文档

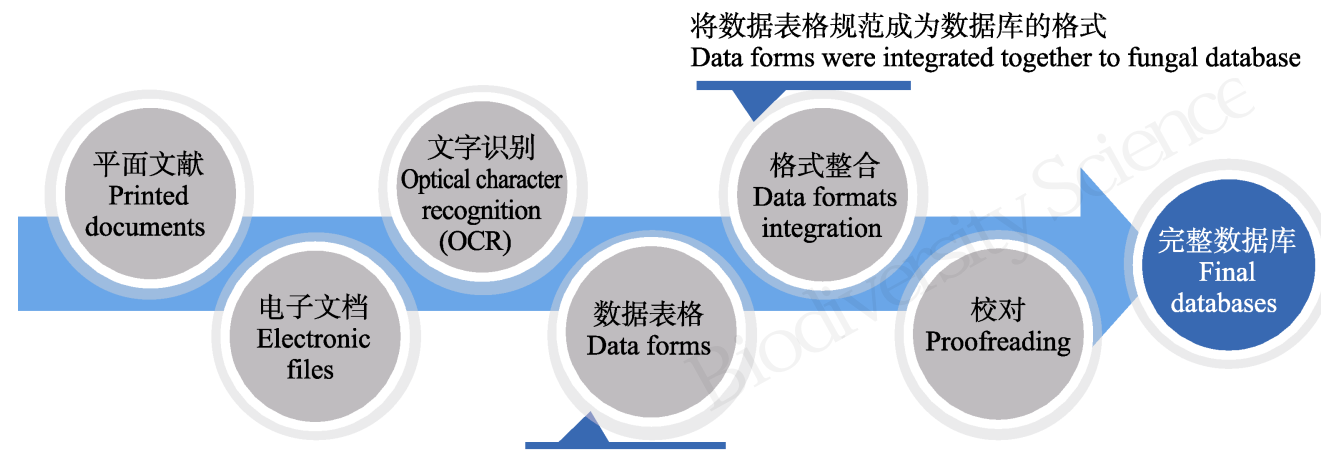

借助数据库软件和编程, 拆分、提取文档中的有效信息, 形成初始数据表格

Data processing using relevant software and programming, transform to the data forms

图1 菌物文献数字化的流程与方法

Fig. 1 Procedures and methods of fungal literature digitization 
的原貌, 同时也把表格、图片等非文本部分完整地 保留下来(姚雪红, 2011)。扫描生成的电子文档均为 图片格式, 仍不便于信息检索与数据挖掘, 需利用 文字识别软件(ABBYY FineReader 11.0)对其进行 格式转换, 文字识别的过程是利用计算机技术与光 学技术, 对印刷或书写的文本图像进行分析处理, 获取其文字及版面信息(寇清华和郑巧红, 2012)。近 年来, 文字识别软件对分辨率较高图像的识别准确 率可达99\%以上(陈杰等，2010)。然而，对于分辨率 较低的电子文档, 文字识别软件的出错率远高于手 工录入和语音输入等人工方法, 因此, 对识别后的 文档进行验证与校对工作十分必要。

校对完成后，将电子文档列入到数字文档库中， 以方便管理和查阅。为了进一步提高文献资源的利 用效率，需对数字文献进行信息挖掘。首先对书籍 或文献的内容和编排格式进行分析, 挖掘其有效信 息，借助文本处理软件 (Notepad++ v7.5.6和Word 2016)和编程软件(Python 3.5), 利用文档的编排规 律, 插入相应的识别符(如文档中未出现过的特殊 符号), 然后使用数据库软件(Access 2016和Excel 2016)读取文档数据, 按识别符分字段写入数据库, 并对数据内容进行编辑处理, 去除冗余数据, 统一 数据格式, 构建出标准、规范的菌物信息数据库。 最后, 将所构建的数据库信息导入中国菌物名录数 据库中, 实现文献资源的有效利用与共享。以《中 国真菌志第十八卷灵芝科》(赵继鼎和张小青, 2000)为例, 根据其书中内容设计和编排格式, 其中 信息可拆分为汉语学名、拉丁名、命名人、异名、 描述、报道地点、世界分布、生境、标本、讨论等 项目, 将其信息分别提取并写入数据库中, 完成对 该书的数字化(数字化示例见附录1)。

按照上述方法, 大型真菌红色名录评估项目组
(以下简称“项目组”)完成了对《中国菌物总汇》、《中 国真菌志》、《真菌名词及名称》等128本纸质菌物 专著的数字化, 为中国菌物名录数据库增添菌物记 录125,556条。数据库扩充之后, 中国菌物名录数据 库中收录了来自372个国内外期刊的7,000多篇论文 和200多部专著的 24 万多条菌物记录信息，涉及中 国分布的菌物物种约2.79万种(Fang et al, 2018)。

\section{数据库在红色名录评估中的应用}

\section{1 数据挖掘与分析的方法}

对于数据库中大量的菌物数据, 项目组构建了 数据分析与挖掘的流程与方法(图2), 以充分利用其 有效信息。首先，针对特定的目标，以数据库为基 础, 根据需求对数据进行关键词篮选, 输出有效数 据。该步骤需要尽可能地扩大选择范围，将可能相 关的数据全部涵盖在内。随后, 对篮选出的数据进 行标准化处理, 使数据栏目格式统一并填补空缺, 便于后续的处理。第三步，对数据进行质量审核, 剔除无用的数据，并修订错误，以确保信息的可靠 性。最后，对审核完成的数据进行分析和处理，根 据需求, 借助数据库软件、编程等方法对数据进行 格式转换、统计分析、自动排版等操作，从而达成 所需目标和结果。

\section{2 评估物种的确定}

此次红色名录所评估的类群是大型真菌。大型 真菌是指能形成肉眼可见的子实体、子座、菌核或 菌体的一类真菌，包括大型子囊菌、大型担子菌和 地衣型真菌等。根据上述特点, 项目组划定的大型 真菌的类群范围: 担子菌门下除黑粉菌纲、锈菌纲、 担子菌酵母以外的类群, 以及子囊菌门下 107 个具 有大型子实体的属和An Enumeration of Lichens in China (Wei, 1991)收录的地衣类群。根据上述类群

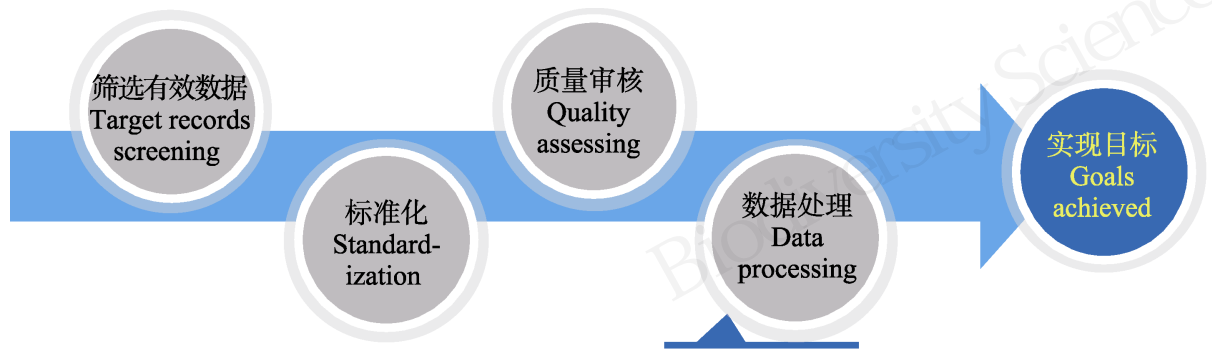

借助数据库软件与编程进行数据格式转换、统计分析, 实现自动排版 Data processing using relevant software and programming, transform to publishing formats

图2 数据挖掘与分析的流程与方法

Fig. 2 Procedures and methods of data mining and analysis 
范围, 依照图 2 的方法从中国菌物名录数据库中筛 选出相关数据, 共提取出大型担子菌记录 117,338 条, 大型子囊菌记录10,213条, 地衣记录37,838条, 涉及菌物名称共14,511 个, 经比对Index Fungorum 数据库, 对物种学名进行核对, 排除有问题的名称 302 个, 去除或合并分类学异名 4,907个, 最终剩余 菌物名称9,302个, 确定为被评估物种。从 Index Fungorum数据库调取这些物种的最新分类地位, 统计 得出评估物种涵盖了我国9,302种大型真菌，其中 大型子囊菌870种, 大型担子菌6,268种, 地衣型真 菌2,164种，分属于2门14纲62目227科1,298属(表1)。

\section{3 菌物汉语学名的选定、修订和新拟}

分析9,302 个评估物种在数据库中的信息发现, 这些物种的汉语学名普遍存在着使用不统一、命名 不规范等问题。其中 6,971 个物种检索到28,200个中 文名称，涉及文献及专著共1,635部/篇，剩余2,331 个物种则没有汉语学名, 需要新拟。为了规范汉语 学名的使用, 项目组以《真菌名词及名称》(中国科 学院微生物研究所, 1986)为命名基准, 参照《真菌、 地衣汉语学名命名法规》(中国菌物学会, 1987), 并 提出了 “汉语学名优先律”, 以解决评估物种的汉语 学名问题。

汉语学名优先律如下(排序靠前的条目具有较 高优先度):

(1) 《真菌名词与名称》中出现的汉语学名。

(2)新组合名的基原名或较早组合名如在《真菌 名词及名称》中出现，其种加词译法应以 《真菌名 词及名称》为准, 除非新组合所在的属中该汉语种 加词已被占用。

(3)除《真菌名词与名称》外, 其他文献中出现 的且符合《真菌、地衣汉语学名命名法规》规定的 汉语学名。

(4)新组合名的基原名或较早组合名如在除《真
菌名词与名称》外的文献中出现, 其种加词译法应 以文献中记载的为准, 除非新组合所在的属中该汉 语种加词已被占用。

(5)新拟汉语学名。根据拉丁名的词根, 特别是 新分类单元发表时所依据的词源学(Etymology), 尽 可能反映属的特征来拟定汉语学名。部分拉丁词根 可参考《真菌名词及名称》的名词部分，但更多的 词根要查阅专门的词典。

针对每一个拉丁学名, 依照以上的优先律顺序 确定其对应的汉语学名。例如：Bovista pusilla (Batsch) Pers.没有找到符合优先律(1)的汉语学名, 但是其基原名Lycoperdon pusillum Batsch出现在《真 菌名词与名称》中, 对应的汉语学名为“小马勃”, 根 据优先律(2)种加词译法“小”被采用, “小”这一译法 在灰球菌属(Bovista)中没有被占用，所以 Bovista pusilla的汉语学名确定为“小灰球菌”。

项目组依照《真菌名词及名称》(中国科学院微 生物研究所, 1986)、《真菌、地衣汉语学名命名法规》 (中国菌物学会, 1987)和“汉语学名优先律”, 借助数 据库软件(Microsoft Office 2016)和程序编写(Python 3.5), 对每一个评估物种的汉语学名进行选定、修订 和新拟工作，同时设置条件进行质量控制，检测每 一个名称是否符合《真菌、地衣汉语学名命名法规》 中提出的命名规则。修订名称示例如下:

“茶薪菇”在多篇文献中均用作拉丁种名Agrocybe chaxingu N. L. Huang的汉语学名(上海农业科学 院食用菌研究所, 1991; 林晓民等, 2005; 张家辉和 邓洪平, 2011), 根据《真菌、地衣汉语学名命名法 规》第6条规则，该名称缺少属主名，而“Agrocybe”的 汉语属名为“田头菇属”(中国科学院微生物研究所, 1986), 故将该种的汉语学名修订为“茶薪田头菇”。

通过上述处理, 9,302个评估物种均有了唯一的 汉语学名, 其中 1,268 个物种的汉语学名来自《真菌

表1 评估的大型真菌类群统计

Table 1 The statistics of assessed macrofungi groups

\begin{tabular}{llllll}
\hline 类群 Groups & 纲 Class & 目 Order & 科 Family & 属 Genus & 种 Species \\
\hline 大型子囊菌 Macro-ascomycetes & 6 & 10 & 33 & 107 & 870 \\
大型担子菌 Macro-basidiomycetes & 3 & 23 & 106 & 841 & 6,268 \\
地衣型真菌 Lichenized fungi $^{\text {总计 Total }}{ }^{*}$ & 9 & 34 & 93 & 352 & 2,164 \\
\hline
\end{tabular}

* 地衣型真菌在属及以上高阶分类单元分别与大型子囊菌和大型担子菌重合, 所以总计的数量为实际分类单元数, 与表中各类群数量之和 不一致。The higher taxa (genus level or higher) of lichenized fungi overlaps with macro-ascomycetes and macro-basidiomycetes respectively, so the Total is the actual number of all taxon, which is inconsistent with the sum of each groups in the table. 
名词及名称》, 2,174 个物种的汉语学名来自 $A n$ Enumeration of Lichenized \& Lichenicolous Fungi in China (Wei, 2018), 3,937个物种的汉语学名选自己 发表的其他文献，其余2,331个物种没有对应的汉 语学名或者汉语学名不符合《真菌、地衣汉语学名 命名法规》, 对其进行了组合和新拟处理, 一共组 合汉语学名 1,140 个, 新拟属名 262 个, 新拟种名 1,191 个, 新拟的汉语学名名单见附录 2 。新拟汉语 学名示例如下:

拉丁种名Agaricus velutinus Pers. 在《真菌名词 及名称》和其他文献中均没有相对应的中文名记载, 根据其种加词的拉丁词根“velutinous”意为“被短线 毛的、丝线状的”, 为其拟定汉语学名“丝线蘑菇”。

\section{4 初评}

本次评估依照“中国大型真菌红色名录评估等 级与标准” (王科等, 2020)展开, 构建了 “初步篎选 归类”的模型方法, 用于大型真菌红色名录的初评 工作。项目组根据中国菌物名录数据库中各评估物 种的地理分布、发表时间、种群变化等信息, 构建 了“初步篮选归类”模型的算法和实施流程(图3), 依 照流程图对9,302种大型菌物评定了初评等级(表2)。 初评结果与最终结果比较发现, 6,524 个评估物种的 最终结果与初评结果相同，占到总数的 $70.14 \%$ 。而 剩余评估等级不一致的2,778个物种中, 发生情况 最多的是由无危调整为数据不足 $(2,628$ 个)。

\section{3 问题及展望}

\section{1 文献数字化的校对问题}

为了保证数字化的电子文档仍与原文档保持 一致, 校对是整个过程最为关键的环节, 需要耗费 大量的时间和人工。除此之外, 文献数字化过程中 的文档扫描、文字识别、信息分类、写入数据库等 步骤都可通过操作计算机完成, 只需少量人工辅助 工作。根据原始文献的分辨率高低，文字识别的错 误率有所不同，大约在 $0.1 \%-5 \%$ 之间。以 《中国真 菌志 第十六卷》(张天宇, 2003)为例, 全书有286页, 共420,000字，其文字识别错误率在 $0.5 \%$ 左右，对其 进行完整的人工校对花费了约 $7 \mathrm{~h}$, 而除此之外的 其他数字化步骤可在 $2 \mathrm{~h}$ 内完成。针对这种情况，或 许从两个方面可以提高校对效率。首先, 改进文字 识别方法，提高文字识别软件的正确率(孙华和张 航, 2010; 邱立松和黄继风, 2013)。其次，提高校对 效率, 借助数据库技术进行自动或半自动校对, 项 目组对于菌物学名、分布地等信息的校对就采取了 这种方案。依照菌物学名和地名的标准清单, 借助 编程和数据库软件操作对本次数字化并写入数据 库的125,556条菌物记录实现了批量比对和订正, 极大地提高了工作效率。

\section{2 菌物汉语学名勘误}

由于菌物汉语学名整理工作步骤繁琐、耗时较

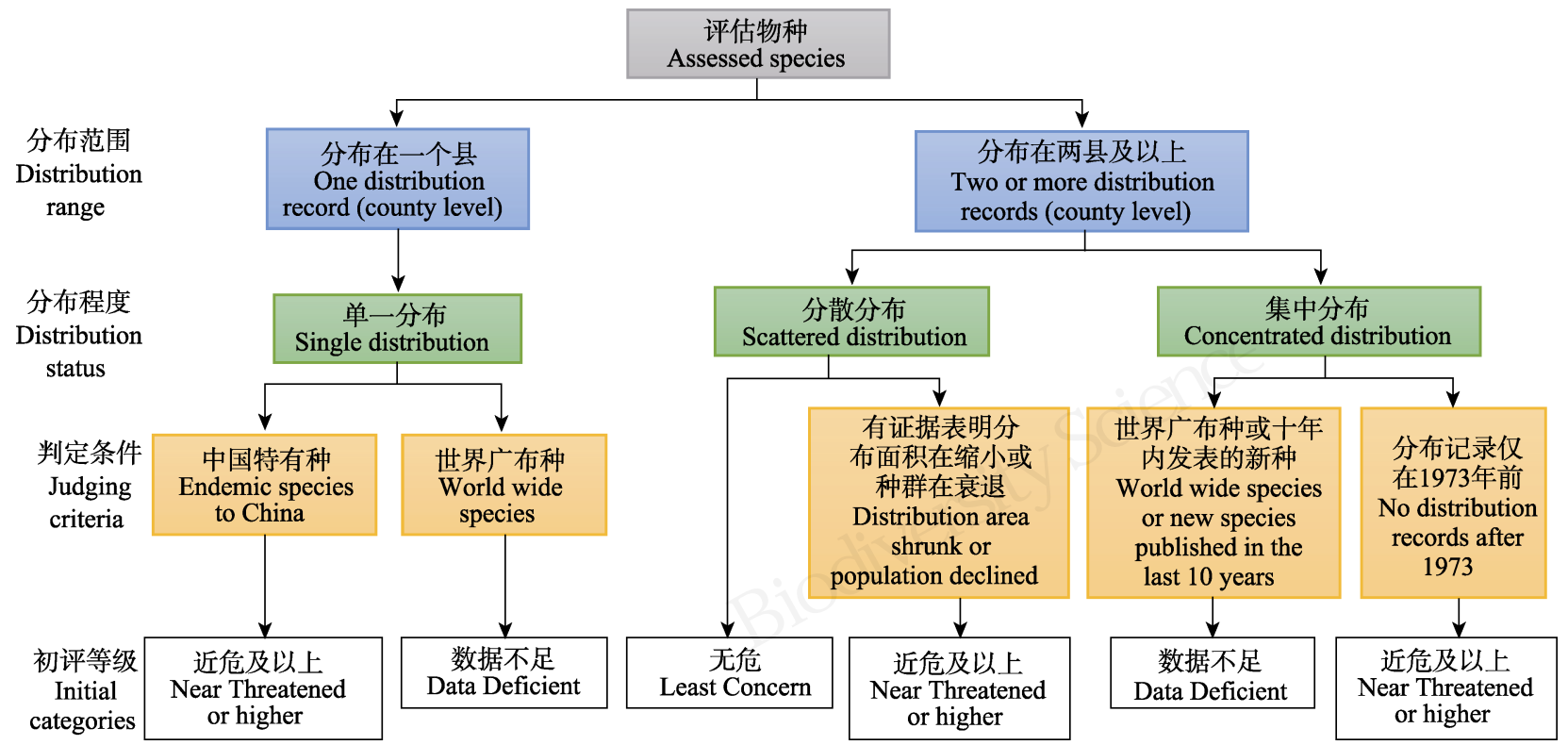

图3中国大型真菌红色名录初步篮选归类方法流程图

Fig. 3 Flowchart of the preliminary screening methods for the red list assessment of macrofungi in China 
表2 中国大型真菌红色名录初评结果与最终结果

Table 2 Preliminary and final results of the red list assessment of macrofungi in China

\begin{tabular}{lll}
\hline 等级 Categories & 初评物种数 Preliminary number of species (\%) & 最终物种数 Final number of species (\%) \\
\hline 灭绝 Extinct (EX) & $0(0.00)$ & $0(0.00)$ \\
野外灭绝 Extinct in the Wild (EW) & $0(0.00)$ & $0(0.00)$ \\
疑似灭绝 Possibly Extinct (PE) & $0(0.00)$ & $1(0.01)$ \\
极危 Critically Endangered (CR) & $0(0.00)$ & $9(0.10)$ \\
濒危 Endangered (EN) & $14(0.15)$ & $25(0.24)$ \\
易危 Vulnerable (VU) & $106(1.14)$ & $62(0.67)$ \\
近危 Near Threatened (NT) & $167(1.80)$ & $101(1.09)$ \\
无危 Least Concern (LC) & $4,925(52.95)$ & $2,764(29.71)$ \\
数据不足 Data Deficient (DD) & $4,090(43.97)$ & $6,340(68.16)$ \\
总数 Total & $9,302(100)$ & $9,302(100)$ \\
\hline
\end{tabular}

长, 难免会出现谬误和疏漏。在整理稿件的过程中, 项目组对《中国生物多样性红色名录一大型真菌 卷》进行了再次核对和修订, 共订正20处, 改动的 名称在附录3中列出。

\section{3 初评结果与最终评估结果的对比}

利用数据库信息的初评结果与最终结果比较 发现(表2), 结果不一致的占到总数的 $29.86 \%$ ，说明 初评的模型和算法还有进一步优化的空间。这可能 是由多方面的因素造成的:

(1)我国菌物资源信息并不充足，90\%以上的评 估物种在数据库中缺乏种群信息和生态情况, 仅仅 有分布报道。分布地的数量在一定程度上可以反映 一个物种的受威胁情况, 但是对于一些地区性特有 种, 了解其在各个分布地的种群情况及栖息地状况 才能更好地进行红色名录评级。比如青藏高原特有 种冬虫夏草(Ophiocordyceps sinensis), 据报道其国 内分布涉及西藏、青海、四川、云南和新疆5个省(自 治区)的100多个分布点( Li et al, 2011), 依照图3所 示模型，其评估等级将落在无危，但是考虑到气候 变化、种群数量等诸多因素, 冬虫夏草最终被评定 为易危(Yan et al, 2017; 李熠等, 2020b)。

(2)中国大型真菌红色名录等级评估标准中涉 及到 5 个类型的 11 条判定标准(王科等, 2020), 而此 次初评模型只使用了其中的一条(B1：分布区)作为 判定依据。其中的根源仍是评估数据不充足造成的, 大量的物种缺乏分布地之外的评估依据。由于初评 的目的是大批量、快速地对评估物种进行分级和归 类, 只能基于现有的较为足够的数据来设计模型。 若能够积极开展大型真菌物种调查监测、加大对分 类学研究的投入, 获取充足的大型真菌物种信息
(李熠等, 2020a), 这一模型方法也会随之得到优化 和完善。

(3)此次初评的方法仅考虑了分布地、特有种等 有限的因素，而忽略了不同类型、不同用途的物种， 这些物种的受威胁因素可能有所不同。一些食药用 菌虽然分布广泛, 但是由于受到人为采挖的影响, 更容易受到威胁。而一些用途不明的物种，往往在 野外无人问津，虽然分布有限，但其在各个分布地 种群数量相对稳定，不易受到威胁。

总的来说，随着评估经验的增加、分类学信息 的积累和数据库的进一步完善, 大型真菌红色名录 的评估方法会更加完善、准确且快速。

致谢: 在汉语学名新拟过程中, 中国科学院微生物 研究所庄文颖院士和庄剑云研究员提出了建设性 意见并修订了部分汉语属名，在此表示衰心感谢！

\section{参考文献}

Chen J, Sun ZG, Zhou SF (2010) Applying image classification using wavelets to digitization of document information. CAAI Transactions on Intelligent Systems, 5, 185-188. (in Chinese with English abstract) [陈杰, 孙忠贵, 周书锋 (2010) 小波的文本图像区分及其在文献信息数字化中的 应用. 智能系统学报, 5, 185-188.]

Edible Fungi Research Institute of Shanghai Academy of Agricultural Sciences (1991) Edible Fungal Flora of China. China Forestry Publishing House, Beijing. (in Chinese) [上 海农业科学院食用菌研究所 (1991) 中国食用菌志.中 国林业出版社，北京.]

Fang R, Kirk P, Wei JC, Li Y, Cai L, Fan L, Wei TZ, Zhao RL, Wang K, Yang ZL, Li TH, Li Y, Phurbu-Dorji, Yao YJ (2018) Country focus: China. In: State of the World's Fungi (ed. Willis KJ), pp. 48-55. Royal Botanic Gardens, Kew. 
Fries EM (1823) Systema Mycologicum. Gryphiswald Mauritius, Lund.

Hawksworth DL, Lucking R (2017) Fungal diversity revisited: 2.2 to 3.8 million species. Microbiology Spectrum, 5(4), 79-80.

Institute of Microbiology, Chinese Academy of Sciences (1986) A Glossary of Terms and Names of Fungi. Science Press, Beijing. (in Chinese) [中国科学院微生物研究所 (1986) 真菌名词及名称. 科学出版社, 北京.]

Kou QH, Zheng QH (2012) Study of the digitalization technologies of documents and their improving directions analysis. Microcomputer Information, 28(5), 109-110, 157. (in Chinese with English abstract) [寇清华, 郑巧红 (2012) 文 献数字化技术的特点及其发展趋势分析. 微计算机信息, 28(5), 109-110, 157.]

Li Y, Liu DM, Wang K, Wu HJ, Cai L, Cai L, Li JS, Yao YJ (2020a) Red list assessment of macrofungi in China: Challenges and measures. Biodiversity Science, 28, 66-73. (in Chinese with English abstract) [李熠, 刘冬梅, 王科, 吴海军, 蔡蕾, 蔡否, 李俊生, 姚一建 (2020a) 中国大型 真菌红色名录评估中存在的问题及今后的对策. 生物多 样性, 28, 66-73.]

Li Y, Tang ZY, Yan YJ, Wang K, Cai L, He JS, Gu S, Yao YJ (2020b) Incorporating species distribution model into the red list assessment and conservation of macrofungi: A case study with Ophiocordyceps sinensis. Biodiversity Science, 28, 99-106. (in Chinese with English abstract) [李熠, 唐志 尧, 问昱晶, 王科, 蔡否, 贺金生, 古松, 姚一建 (2020b) 物种分布模型在大型真菌红色名录评估及保护中的应用: 以冬虫夏草为例. 生物多样性, 28, 99-106.]

Li Y, Wang XL, Jiao L, Jiang Y, Li H, Jiang SP, Lhosumtseiring N, Fu SZ, Dong CH, Zhan Y, Yao YJ (2011) A survey of the geographic distribution of Ophiocordyceps sinensis. Journal of Microbiology, 49, 913-919.

Lin XM, Li ZQ, Hou J (2005) The Diversity of Macrofungi in China. China Agriculture Press, Beijing. (in Chinese) [林晓 民，李振歧，侯军 (2005) 中国大型真菌的多样性. 中国 农业出版社, 北京.]

McNeil J, Turland NJ (2011) Major changes to the Code of Nomenclature-Melbourne, July 2011. Taxon, 60, 1495-1497.

Mycological Society of China (1987) Nomenclature Code of Fungal Chinese Names. Acta Mycologica Sinica, 6, 61-64. (in Chinese) [中国菌物学会 (1987) 真菌、地衣汉语学名 命名法规. 真菌学报, 6, 61-64.]

Qiu LS, Huang JF (2013) Feature extraction and recognition of information in document image. Computer \& Digital Engineering, 41, 1981-1984. (in Chinese with English abstract) [邱立松, 黄继风 (2013) 文本图像信息的提取与识别. 计算机与数字工程, 41, 1981-1984.]

Saccardo PA (1898) Sylloge Fungorum: Omnium Hucusque Cognitorum. Lipsiae Fratres Borntraeger, New York.

Sun H, Zhang H (2010) Survey on Chinese character recognition method. Computer Engineering, 36(20), 194-197. (in Chinese with English abstract) [孙华, 张航 (2010) 汉字识 别方法综述. 计算机工程, 36(20), 194-197.]
Tai FL (1979) Sylloge Fungorum Sinicorum. Science Press, Beijing. (in Chinese) [戴芳澜 (1979) 中国真菌总汇. 科学 出版社, 北京.]

Teng SC (1963) Fungi of China. Science Press, Beijing. (in Chinese) [邓叔群 (1963) 中国的真菌. 科学出版社, 北 京.]

Turland NJ, Wiersema JH, Barrie FR, Greuter W, Hawksworth DL, Herendeen PS, Knapp S, Kusber WH, Li DZ, Marhold K, May TW, McNeill J, Monro AM, Prado J, Price MJ, Smith GF (2018) International Code of Nomenclature for Algae, Fungi, and Plants (Shenzhen Code) adopted by the Nineteenth International Botanical Congress Shenzhen, China, July 2017. Regnum Vegetabile 159. Glashütten: Koeltz Botanical Books. https://doi.org/10.12705/Code.2018.

Wang K, Liu DM, Cai L, Wu HJ, Li Y, Wei TZ, Wang YH, Wu HM, Wei XD, Li BB, Li JS, Yao YJ (2020) Methods and procedures of the red list assessment of macrofungi in China. Biodiversity Science, 28, 11-19. (in Chinese with English abstract) [王科, 刘冬梅, 蔡蕾, 吴海军, 李熠, 魏 铁铮, 王永会, 吴红梅, 卫晓丹, 李斌斌, 李俊生, 姚一 建 (2020) 中国大型真菌红色名录评估方法和程序. 生 物多样性, 28, 11-19.]

Wei JC (1991) An Enumeration of Lichens in China. International Academic Publishers, Beijing.

Wei JC (2018) An Enumeration of Lichenized \& Lichenicolous Fungi in China. China Forestry Publishing House, Beijing.

Yan YJ, Li Y, Wang WJ, He JS, Yang RH, Wu HJ, Wang XL, Jiao L, Tang ZY, Yao YJ (2017) Range shifts in response to climate change of Ophiocordyceps sinensis, a fungus endemic to the Tibetan Plateau. Biological Conservation, 206, 143-150.

Yao XH (2011) Practice and exploration of the literature digitalization. Information Science, 29, 1838-1840. (in Chinese with English abstract) [姚雪红 (2011) 文献数字化的实践 与探索. 情报科学, 29, 1838-1840.]

Zhang JH, Deng HP (2011) Primary Color Atlas of Fungi in Jinyun Mountain. Southwest Normal University Press, Chongqing. (in Chinese) [张家辉, 邓洪平 (2011) 缙云山草 菌原色图集. 西南师范大学出版社, 重庆.]

Zhang TY (2003) Flora Fungorum Sinicorum, Vol. 16, Alternaria. Science Press, Beijing. (in Chinese) [张天宇 (2003) 中国真菌志第十六卷 链格狍属. 科学出版社, 北京.]

Zhao JD, Zhang XQ (2000) Flora Fungorum Sinicorum, Vol. 18, Ganodermataceae. Science Press, Beijing. (in Chinese) [赵继鼎, 张小青 (2000) 中国真菌志 第十八卷 灵芝科. 科学出版社, 北京.]

Zheng RY, Wei JC, Hu HJ, Yu YN, Wu PC, Xing GX, Liu B (1990) A Glossary of Terms and Names of Cryptogamia. Science Press, Beijing. (in Chinese) [郑儒永, 魏江春, 胡 鸿钧, 余永年, 吴鹏程, 邢公侠, 刘波 (1990) 孢子植物 名词及名称. 科学出版社, 北京.]

(责任编委：杨祝良 责任编辑：问文杰) 
附录1 《中国真菌志 第十八卷 灵芝科》数字化示例

Appendix 1 Digitized example of Flora Fungorum Sinicorum (Vol. 18): Ganodermataceae

\section{茶病灵芝图 41}

Ganoderma theaecolum J. D. Zhao, Acta Mycol. Sinica 3 (1): 16, Fig. 2, Pl. I-2, 1984; Zhao, The Ganodermataceae in China (Chinese edition). p. 93, Fig. 32, 1989. 担子果一年生, 无柄或具短柄, 木栓质到木质。菌盖半圆形或近扇形, 表面红褐色 到紫褐色, 具很强的似漆样光泽, 有时光泽稍弱, 10-14 ×6-8cm, 厚 7-15 mm; 边 缘与菌盖同色或呈淡黄白色, 具不显著的宽同心环沟, 平滑或钝, 下面不芓; 菌肉厚 4-8mm, 分层不明显, 上层淡白褐色, 下层淡褐色到褐色; 茠管长 $3-7 \mathrm{~mm}$, 淡褐色 到褐色; 孔面污白色、淡黄色或黄色; 管口略圆形或近角形, 每毫米 4-5 个。

皮壳构造呈不规则的拟子实层型, 淡黄褐色, 组成菌丝棍棒状, 顶端膨大部分宽 5-7 $\mu \mathrm{m}$, 长 $25-30 \mu \mathrm{m}$, 易与菌肉组织分离。

菌丝系统三体型: 生殖菌丝无色透明, 薄壁, 直径 3-4 $\mu \mathrm{m}$; 骨架菌丝淡黄褐色到 浅褐色, 厚壁到实心, 树状分枝, 骨架干直径 4-5 $\mu \mathrm{m}$, 分枝末端形成鞭毛状无色缠绕 菌丝; 缠绕菌丝无色, 厚壁, 分枝, 弯曲, 直径 $1-2 \mu \mathrm{m}$ 。

担狍子稀少, 卵圆形或顶端平截, 双层壁, 外壁无色透明, 平滑, 内壁淡黄褐色, 小刺稀少或不清楚, 7一 $9 \times 5.2-6.2 \mu \mathrm{m}$ 。

模式产地: 中国 (海南)。

生境: 生茶树根部, 引起茶树红根病。有时生台湾相思树上。

研究标本: 湖北应山 (37909); 海南琼中县 (42788 模式)、临高县 (42785)、海 口市 (47540)、尖峰岭 (37881); 广西弄岗（42786); 云南下关 (48243); 香港 $(62560)$ 。

世界分布: 中国。

讨论: 此种的特点是引起茶树红根病。该种担子果无柄, 具有很强的似漆样光泽。 菌肉分层, 但层次不清楚。它与 G. lucidum 相近似, 但后者担孢子较大, 可达 8.5$11.2(-12.1) \times 5.2-6.9 \mu \mathrm{m}$ 。通常具有较长的菌柄, 并有类灰球菌型的缠绕菌丝。

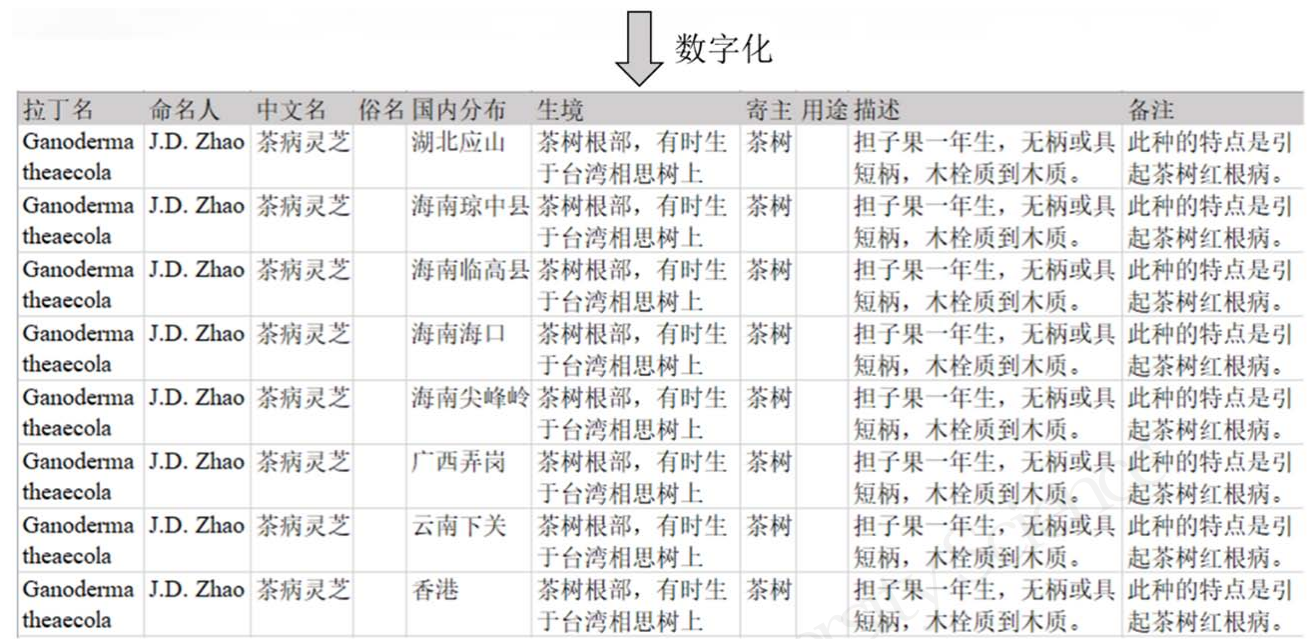


附录2《中国生物多样性红色名录一一型真菌卷》中新拟汉语学名名单

Appendix 2 List of new fungal Chinese names in Red List of China's Biodiversity-Macrofungi

\begin{tabular}{|c|c|c|c|}
\hline 拉丁学名 & 汉语学名 & 拉丁学名 & 汉语学名 \\
\hline Abundisporus mollissimus B. K. Cui \& C. L. Zhao & 软多狍孔菌 & Aleurina nigrodisca Sawada & 黑粉盘菌 \\
\hline Acanthobasidium penicillatum (Burt) Sheng H. Wu & 帚状刺担菌 & Aleurodiscus botryosus Burt & 葡萄盘革菌 \\
\hline $\begin{array}{l}\text { Acanthofungus rimosus Sheng H. Wu, Boidin \& } \\
\text { C. Y. Chien }\end{array}$ & 裂纹刺囊革菌 & Aleurodiscus cremicolor Hjortstam \& Ryvarden & 奶油色盘革菌 \\
\hline Agaricus amanitiformis Wasser & 鹅膏状蘑菇 & Aleurodiscus diffissus (Sacc.) Burt & 罕见盘革菌 \\
\hline Agaricus angustus Pers. & 狭蘑菇 & Amanita anisata (Murrill) Murrill & 异形鹅膏 \\
\hline Agaricus bisporatus Contu & 二狍蘑菇 & Amanita australis G. Stev. & 南方鹅膏 \\
\hline Agaricus brunneolus (J. E. Lange) Pilát & 褐蘑菇 & Amanita baccata (Fr.) Gillet & 浆果鹅膏 \\
\hline Agaricus chaetodes Berk. \& M. A. Curtis & 毛蘑菇 & Amanita bivolvata Peck & 双托鹅膏 \\
\hline Agaricus cladophyllus Lév. & 枝生蘑菇 & Amanita cheelii P. M. Kirk & 朱氏鹅膏 \\
\hline Agaricus compernis Fr. & 康珀蘑菇 & Amanita cothurnata G. F. Atk. & 具鞘鹅膏 \\
\hline Agaricus cupreobrunneus (Jul. Schäff. \& Steer) Pilát & 铜褐蘑菇 & Amanita puella Gonn. \& Rabenh. & 美鹅膏 \\
\hline Agaricus dactyliotus Berk. \& Mont. & 枝蘑菇 & Amanita spadicea Pers. & 菄褐鹅膏 \\
\hline Agaricus depauperatus (F. H. Møller) Pilát & 萎缩蘑菇 & Amanita verrucosa Lam. & 多疮鹅膏 \\
\hline Agaricus fabaceus Berk. & 虫豆蘑菇 & Amauroderma insulare (Har. \& Pat.) Torrend & 岛乌芝 \\
\hline $\begin{array}{l}\text { Agaricus flocculosipes R. L. Zhao, Desjardin, Guinb. \& } \\
\text { K. D. Hyde }\end{array}$ & 卷毛蘑菇 & Amauroderma perplexum Corner & 惑乌芝 \\
\hline Agaricus glaucobrunneus Berk. \& M. A. Curtis & 绿褐蘑菇 & $\begin{array}{l}\text { Amylocorticiellum cremeoisabellinum (Litsch.) } \\
\text { Spirin \& Zmitr. }\end{array}$ & $\begin{array}{l}\text { 浅黄小淀粉伏 } \\
\text { 革菌 }\end{array}$ \\
\hline $\begin{array}{l}\text { Agaricus guizhouensis Y. Gui, Zuo Y. Liu \& } \\
\text { K. D. Hyde }\end{array}$ & 贵州蘑菇 & Amylocorticium indicum K. S. Thind \& S. S. Rattan & 印度淀粉伏革菌 \\
\hline Agaricus hondensis Murrill & 本田蘑菇 & Amylocorticium subincarnatum (Peck) Pouzar & $\begin{array}{l}\text { 亚肉质淀粉伏 } \\
\text { 革菌 }\end{array}$ \\
\hline $\begin{array}{l}\text { Agaricus longistipes Y. Gui, Zuo Y. Liu, Callac, } \\
\text { L. A. Parra \& K. D. Hyde }\end{array}$ & 长柄蘑菇 & Amylocorticium subsulphureum (P. Karst.) Pouzar & $\begin{array}{l}\text { 亚硫色淀粉伏 } \\
\text { 革菌 }\end{array}$ \\
\hline Agaricus macrosporoides Bohus & 似大孢蘑菇 & $\begin{array}{l}\text { Amylosporus rubellus (Y. C. Dai) Y. C. Dai, } \\
\text { Jia J. Chen \& B. K. Cui }\end{array}$ & 红粉孢菌 \\
\hline $\begin{array}{l}\text { Agaricus megalocarpus Y. Gui, Zuo Y. Liu, Callac, } \\
\text { L. A. Parra \& K. D. Hyde }\end{array}$ & 大果蘑菇 & Amylosporus ryvardenii Stalpers & 吕氏粉孢菌 \\
\hline Agaricus minimus (Ricken) Pilát & 迷你蘑菇 & Anellaria ochroleuca Sawada & 黄白斑褶伞 \\
\hline Agaricus ochraceosquamulosus Heinem. & 红褐鳞蘑菇 & Anellaria planiuscula Sawada & 平斑褶伞 \\
\hline Agaricus perturbans E. Ludw. \& W. Pohl & 扰乱蘑菇 & $\begin{array}{l}\text { Anthracophyllum lateritium } \\
\text { (Berk. \& M. A. Curtis) Singer }\end{array}$ & 砖红炭褶菌 \\
\hline Agaricus phaeolepidotus F. H. Møller & 暗鳞蘑菇 & Antrodia lalashana T. T. Chang \& W. N. Chou & 拉拉山薄孔菌 \\
\hline Agaricus pocillator Murrill & 莲座蘑菇 & $\begin{array}{l}\text { Antrodia mappa (Overh. \& J. Lowe) Miettinen \& } \\
\text { Vlasák }\end{array}$ & 地貌状薄孔菌 \\
\hline Agaricus poigenus Berk. \& M. A. Curtis & 早熟禾蘑菇 & Antrodiella nanospora H. S. Yuan & 微孢小薄孔菌 \\
\hline Agaricus pseudopratensis (Bohus) Wasser & 拟草地蘑菇 & Antrodiella pendulina H. S. Yuan & 下垂小薄孔菌 \\
\hline Agaricus spodophyllus Krombh. & 灰褶蘑菇 & Antrodiella ussurii Y. C. Dai \& Niemelä & 乌苏里小薄孔菌 \\
\hline Agaricus velutinus Pers. & 丝绒蘑菇 & Aphanobasidium pseudotsugae (Burt) Boidin \& Gilles & 假铁杉丝瑚菌 \\
\hline Agaricus xantholepis F. H. Møller & 黄鳞蘑菇 & Armillaria subcava (Schumach.) Sacc. & 亚空蜜环菌 \\
\hline Agrocybe firma (Peck) Singer & 紧实田头菇 & $\begin{array}{l}\text { Arrhenia discorosea (Pilát) Zvyagina, Aleks. \& } \\
\text { Bulyonk. }\end{array}$ & 分离健孔菌 \\
\hline Albatrellopsis flettii (Morse ex Pouzar) Audet & 蓝黄拟地花菌 & Arrhenia lobata (Pers.) Kühner \& Lamoure ex Redhead & 1裂片健孔菌 \\
\hline Albatrellus citrinus Ryman & 橘黄地花菌 & Artomyces cristatus (Kauffman) Jülich & 冠密瑚菌 \\
\hline Albatrellus jianfenglingensis G. Y. Zheng & 尖峰岭地花菌 & Ascobolus scatigenus (Berk. \& M. A. Curtis) Brumm. & 寡纹粪盘菌 \\
\hline Aleuria bicucullata Boud. & 双勺网孢盘菌 & Ascocoryne trichophora (A. L. Sm.) Seifert & 产丝胞囊盾菌 \\
\hline
\end{tabular}


附录2 (续) Appendix 2 (continued)

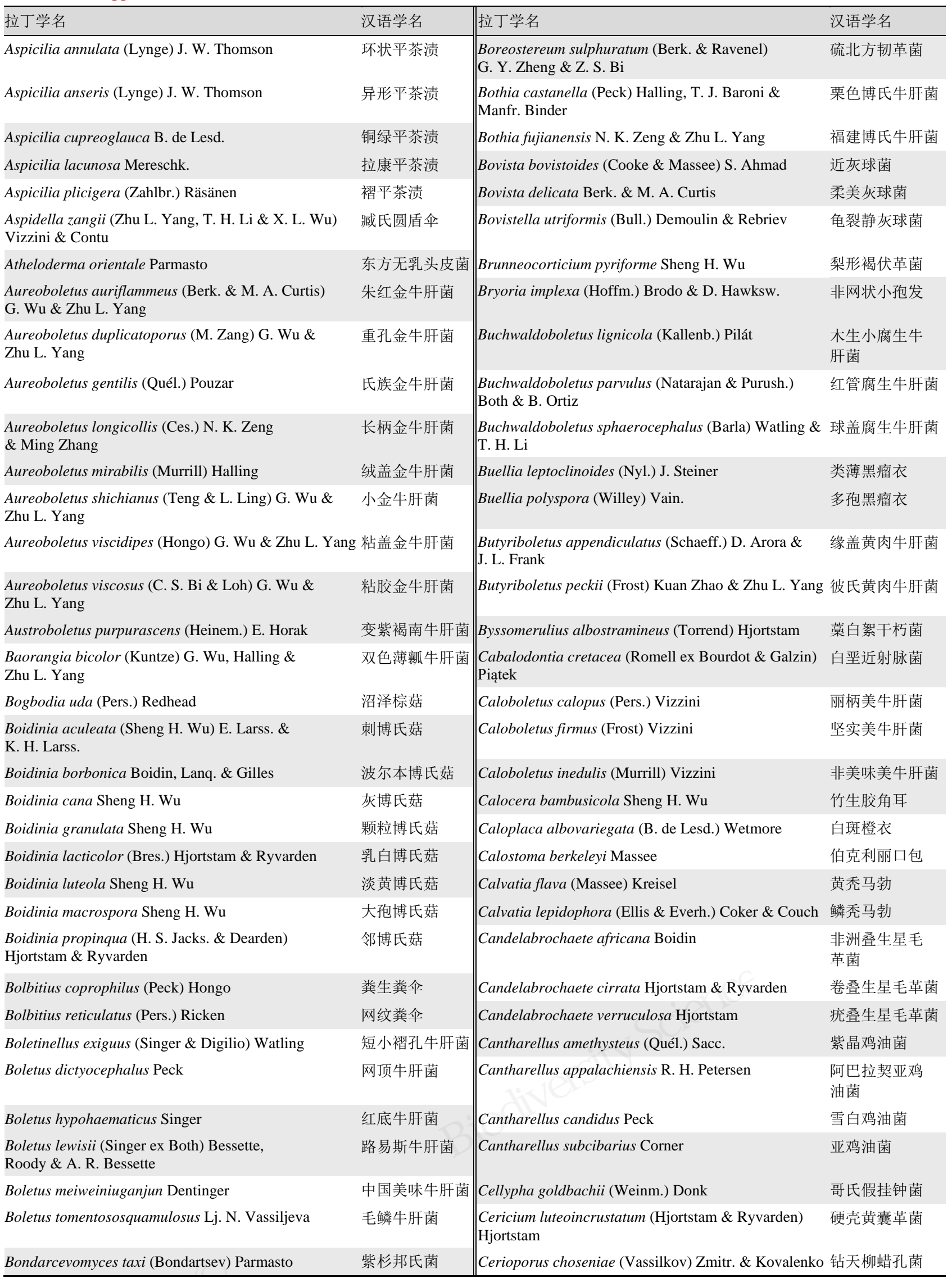


附录2 (续) Appendix 2 (continued)

\begin{tabular}{|c|c|c|c|}
\hline 拉丁学名 & 汉语学名 & 拉丁学名 & 汉语学名 \\
\hline Ceriporia davidii (D. A. Reid) M. Pieri \& B. Rivoire & 大卫蜡卧孔菌 & $\begin{array}{l}\text { Clitocybula lignicola (Lj. N. Vassiljeva) } \\
\text { E. F. Malysheva \& O. V. Morozova }\end{array}$ & 木生小杯伞 \\
\hline Ceriporia jiangxiensis B. S. Jia \& B. K. Cui & 江西蜡卧孔菌 & Collybia cirrhata (Schumach.) Quél. & 卷金钱菌 \\
\hline Ceriporiopsis lavendula B. K. Cui & 薰衣草拟蜡孔菌 & Collybia velutinopunctata $\mathrm{Lj}$. N. Vassiljeva & 丝线金钱菌 \\
\hline Cerocorticium molle (Berk. \& M. A. Curtis) Jülich & 莫尔蜡伏革菌 & Coltricia spina Y. C. Dai & 刺集毛菌 \\
\hline Cerrena aurantiopora J. S. Lee \& Y. W. Lim & $\begin{array}{l}\text { 金黄狍下皮黑 } \\
\text { 孔菌 }\end{array}$ & Conferticium ochraceum (Fr.) Hallenb. & 㬨黄集革菌 \\
\hline Chaenothecopsis hunanensis Rikkinen \& Tuovila & 湖南类口果粉衣 & Coniophora fusispora (Cooke \& Ellis) Cooke & 梭狍粉孢革菌 \\
\hline Chaenothecopsis khayensis Rikkinen \& Tuovila & $\begin{array}{l}\text { 非洲楝类口果 } \\
\text { 粉衣 }\end{array}$ & Conocybe aberrans (Kühner) Kühner & 畸雉盖伞 \\
\hline Chaenothecopsis perforata Rikkinen \& Tuovila & 穿孔类口果粉衣| & Conocybe brunnea J. E. Lange \& Kühner ex Watling & 褐雉盖伞 \\
\hline Chaenothecopsis resinophila Rikkinen \& Tuovila & 胶类口果粉衣 & Conocybe cyanopus (G. F. Atk.) Kühner & 暗蓝雉盖伞 \\
\hline Cheilymenia vitellina (Pers.) Dennis & 蛋黄缘刺盘菌 & Conocybe fibrillosipes Watling & 纤丝雉盖伞 \\
\hline Chlorophyllum alborubescens (Hongo) Vellinga & 变红青褶伞 & Conocybe filaris (Fr.) Kühner & 线雉盖伞 \\
\hline $\begin{array}{l}\text { Chlorophyllum sphaerosporum Z. W. Ge \& } \\
\text { Zhu L. Yang }\end{array}$ & 球狍青褶伞 & Conocybe incarnata (Jul. Schäff.) Hauskn. \& Arnolds & 肉色雉盖伞 \\
\hline $\begin{array}{l}\text { Chromosera cyanophylla (Fr.) Redhead, Ammirati \& } \\
\text { Norvell }\end{array}$ & 蓝紫褶菇 & Conocybe mesospora Kühner ex Watling & 中孢雉盖伞 \\
\hline Chroogomphus sibiricus (Singer) O. K. Mill. & 西伯利亚色钉菇 & Conocybe mutabilis Watling & 多变雉盖伞 \\
\hline Cladonia luteoalba A. Wilson \& Wheldon & 黄白石荵 & Conocybe pilosella (Pers.) Kühner & 线毛雉盖伞 \\
\hline Cladonia magyarica Vain. ex Gyeln. & 鳞杯石荵 & Conocybe rickeniana P. D. Orton & 里肯雉盖伞 \\
\hline Cladonia melanocaulis S. Stenroos & 黑柄石荵 & Conocybe semiglobata Kühner \& Watling & 半圆雉盖伞 \\
\hline Clavaria flavipes Pers. & 黄珊瑚菌 & $\begin{array}{l}\text { Conocybe siennophylla (Berk. \& Broome) Singer } \\
\text { ex Chiari \& Papetti }\end{array}$ & 赫叶雉盖伞 \\
\hline Clavaria gelatinosa Coker & 胶质珊瑚菌 & Conocybe utriformis P. D. Orton & 龟裂雉盖伞 \\
\hline Clavaria paludicola Lib. & 沼生珊瑚菌 & $\begin{array}{l}\text { Coprinellus flocculosus (DC.) Vilgalys, } \\
\text { Hopple \& Jacq. Johnson }\end{array}$ & 卷毛小鬼伞 \\
\hline Clavaria rubicundula Leathers & 深红珊瑚菌 & \begin{tabular}{|l} 
Coprinellus heptemerus (M. Lange \& A. H. Sm.) \\
Vilgalys, Hopple \& Jacq. Johnson
\end{tabular} & 无锁小鬼伞 \\
\hline Clavaria spiculospora G. F. Atk. & 尖狍珊瑚菌 & \begin{tabular}{|l} 
Coprinellus marculentus (Britzelm.) Redhead, \\
Vilgalys \& Moncalvo
\end{tabular} & 灰糠小鬼伞 \\
\hline Clavariadelphus americanus (Corner) Methven & 美洲棒瑚菌 & Coprinopsis alopecia (Lasch) La Chiusa \& Boffelli & 秃拟鬼伞 \\
\hline Clavicorona taxophila (Thom) Doty & 紫杉冠瑚菌 & $\begin{array}{l}\text { Coprinopsis jonesii (Peck) Redhead, } \\
\text { Vilgalys \& Moncalvo }\end{array}$ & 琼斯拟鬼伞 \\
\hline $\begin{array}{l}\text { Clavulicium macounii (Burt) J. Erikss. \& Boidin ex } \\
\text { Parmasto }\end{array}$ & 梅孔似锁瑚菌 & $\begin{array}{l}\text { Coprinopsis lagopides (P. Karst.) Redhead, Vilgalys \& } \\
\text { Moncalvo }\end{array}$ & 白毛拟鬼伞 \\
\hline Clavulina castaneipes (G. F. Atk.) Corner & 栗色锁瑚菌 & Coprinopsis melanthina (Fr.) Örstadius \& E. Larss. & 黑拟鬼伞 \\
\hline $\begin{array}{l}\text { Clavulinopsis laeticolor (Berk. \& M. A. Curtis) } \\
\text { R. H. Petersen }\end{array}$ & 悦色拟锁瑚菌 & $\begin{array}{l}\text { Coprinopsis narcotica (Batsch) Redhead, Vilgalys \& } \\
\text { Moncalvo }\end{array}$ & 麻醉拟鬼伞 \\
\hline Clavulinopsis umbrinella (Sacc.) Corner & 赫色拟锁瑚菌 & $\begin{array}{l}\text { Coprinopsis neolagopus (Hongo \& Sagara) Redhead, } \\
\text { Vilgalys \& Moncalvo }\end{array}$ & 新白绒拟鬼伞 \\
\hline $\begin{array}{l}\text { Clitocella fallax (Quél.) Kluting, T. J. Baroni \& } \\
\text { Bergemann }\end{array}$ & 假灰红褶菌 & $\begin{array}{l}\text { Coprinopsis novorugosobispora Fukiharu \& } \\
\text { Yamakoshi }\end{array}$ & 双狍拟鬼伞 \\
\hline Clitocybe fasciculata H. E. Bigelow \& A. H. Sm. & 簇生杯伞 & $\begin{array}{l}\text { Coprinopsis stercorea (Fr.) Redhead, Vilgalys \& } \\
\text { Moncalvo }\end{array}$ & 粪生拟鬼伞 \\
\hline Clitocybe fuscosquamula J. E. Lange & 暗鳞杯伞 & Coprinus ornatus Copel. & 纹饰鬼伞 \\
\hline Clitocybe hydrogramma (Bull.) P. Kumm. & 湿纹杯伞 & Coprinus squamosus Morgan & 鳞鬼伞 \\
\hline Clitocybe strigosa Harmaja & 硬毛杯伞 & Cordyceps coccinea Penz. \& Sacc. & 绯红虫草 \\
\hline Clitocybe subalutacea (Batsch) P. Kumm. & 亚淡黄杯伞 & Cordyceps deflectens Penz. \& Sacc. & 弯曲虫草 \\
\hline Clitocybe subinvoluta (Batsch) Sacc. & 亚内卷杯伞 & Cordyceps flavobrunnescens Henn. & 棕黄虫草 \\
\hline
\end{tabular}


附录2 (续) Appendix 2 (continued)

\begin{tabular}{|c|c|c|c|}
\hline 拉丁学名 & 汉语学名 & 拉丁学名 & 汉语学名 \\
\hline Cordyceps lacroixii Har. \& Pat. & 拉克虫草 & Cortinarius sphaerospermus Kauffman & 球子丝膜菌 \\
\hline Cordyceps scottianus Olliff & 斯科特虫草 & Cortinarius splendens Rob. Henry & 光泽丝膜菌 \\
\hline Cordyceps variegata Moureau & 彩斑虫草 & Cortinarius sublatisporus Svrček & 亚宽孢丝膜菌 \\
\hline Coronicium proximum (H. S. Jacks.) Jülich & 近轴冕瑚菌 & Cortinarius triumphans Fr. & 凯旋丝膜菌 \\
\hline Corticium rubrocanum Thüm. & 红伏革菌 & Cortinarius umbrinolens P. D. Orton & 茶褐丝膜菌 \\
\hline Cortinarius alnetorum (Velen.) M. M. Moser & 赤杨丝膜菌 & Cortinarius urbicus (Fr.) Fr. & 城市丝膜菌 \\
\hline Cortinarius alneus M. M. Moser & 桤木丝膜菌 & Cortinarius violaceo-olivaceus M. M. Moser & 紫绿丝膜菌 \\
\hline Cortinarius argenteopileatus Nezdojm. & 银盖丝膜菌 & Cortinarius xantho-ochraceus P. D. Orton & 黄橄榄色丝膜菌 \\
\hline Cortinarius balaustinus Fr. & 石榴丝膜菌 & Cotylidia pannosa (Sowerby) D. A. Reid & 毡状杯革菌 \\
\hline Cortinarius betulinus J. Favre & 桦生丝膜菌 & Cotylidia undulata (Fr.) P. Karst. & 波状杯革菌 \\
\hline Cortinarius candelaris Fr. & 烛丝膜菌 & Craterellus minimus Saut. & 微喇叭菌 \\
\hline Cortinarius cereifolius (M. M. Moser) M. M. Moser & 蜡叶丝膜菌 & Crepidotus carpaticus Pilát & 喀尔巴阶靴耳 \\
\hline Cortinarius colus Fr. & 共同丝膜菌 & Crepidotus caspari Velen. & 卡斯珀靴耳 \\
\hline $\begin{array}{l}\text { Cortinarius crassifolius (Velen.) Kühner \& Romagn. } \\
\text { ex Bon }\end{array}$ & 厚丝膜菌 & Crepidotus citrinus Petch & 柠檬黄靴耳 \\
\hline Cortinarius croceus (Schaeff.) Gray & 杏黄丝膜菌 & Crepidotus croceotinctus Peck & 杏黄靴耳 \\
\hline Cortinarius crystallinus Fr. & 晶体丝膜菌 & Crepidotus latifolius Peck & 广叶靴耳 \\
\hline Cortinarius cyanites Fr. & 暗蓝丝膜菌 & Crepidotus stipitatus Kauffman & 柄靴耳 \\
\hline Cortinarius decoratus Bataille & 纹饰丝膜菌 & Crinipellis iopus Singer & 紫罗兰毛皮伞 \\
\hline Cortinarius dilutus (Pers.) Fr. & 淡色丝膜菌 & Crinipellis piceae Singer & 云杉毛皮伞 \\
\hline Cortinarius elegantissimus Rob. Henry & 美丽丝膜菌 & Crinipellis setipes (Peck) Singer & 刚毛毛皮伞 \\
\hline Cortinarius fasciatus Fr. & 簇生丝膜菌 & Crucispora sinensis & 中国十字狍伞 \\
\hline Cortinarius flexipes (Pers.) Fr. & 弯柄丝膜菌 & Crustoderma dryinum (Berk. \& M. A. Curtis) Parmasto & 栋壳皮革菌 \\
\hline Cortinarius glaucopus (Schaeff.) Gray & 蓝绿丝膜菌 & Crustomyces expallens (Bres.) Hjortstam & 苍白壳革菌 \\
\hline Cortinarius helvolus (Bull.) Fr. & 赫黄丝膜菌 & Cudonia convoluta Yasuda & 卷地锤菌 \\
\hline Cortinarius illibatus Fr. & 狭丝膜菌 & Cunninghammyces umbonatus (G. Cunn.) Stalpers & 脐突坎氏菌 \\
\hline Cortinarius incisus (Pers.) Fr. & 锐裂丝膜菌 & Cyathia rufipes (Ellis \& Everh.) V. S. White & $\begin{array}{l}\text { 隆纹间型黑蛋 } \\
\text { 巢菌 }\end{array}$ \\
\hline Cortinarius livor Fr. & 肝色丝膜菌 & Cyclocybe parasitica (G. Stev.) Vizzini & 寄生环伞 \\
\hline Cortinarius ochroleucus (Schaeff.) Fr. & 淡赫丝膜菌 & Cycloderma indicum Klotzsch & 印度环皮包 \\
\hline Cortinarius olympianus A. H. Sm. & 奥林匹亚丝膜菌 & \begin{tabular}{|l} 
Cystidiodontia isabellina (Berk. \& Broome) \\
Hjortstam \& Ryvarden
\end{tabular} & 古铜囊齿革菌 \\
\hline Cortinarius polymorphus Rob. Henry & 复型丝膜菌 & \begin{tabular}{|l} 
Cystidiodontia laminifera (Berk. \& M. A. Curtis) \\
Hjortstam
\end{tabular} & 薄囊齿革菌 \\
\hline Cortinarius porphyropus (Alb. \& Schwein.) Fr. & 岩红柄丝膜菌 & Cystodermella australis (A. H. Sm. \& Singer) Vizzini & 南方小囊皮菌 \\
\hline Cortinarius pseudovariicolor Damblon \& Lambinon & 假变色丝膜菌 & Cystodermella japonica (Thoen \& Hongo) Harmaja & 日本小囊皮菌 \\
\hline Cortinarius rickenianus Maire & 里肯丝膜菌 & Cystodermella terryi (Berk. \& Broome) Bellù & 特里小囊皮菌 \\
\hline Cortinarius rigens (Pers.) Fr. & 刚丝膜菌 & Cystolepiota adulterina (F. H. Møller) Bon & 小鳞囊小伞 \\
\hline Cortinarius rubellus Cooke & 微红丝膜菌 & Cystolepiota eriophora (Peck) Knudsen & 刺囊小伞 \\
\hline Cortinarius rubricosus (Fr.) Fr. & 红肉丝膜菌 & |Cystolepiota hetieri (Boud.) Singer & 哈氏囊小伞 \\
\hline Cortinarius russeus Rob. Henry & 锈红丝膜菌 & Cystolepiota hetieriana (Locq.) Bresinsky \& H. Haas & 类哈氏囊小伞 \\
\hline Cortinarius saturninus (Fr.) Fr. & 土星丝膜菌 & Cystolepiota moelleri Knudsen & 穆勒囊小伞 \\
\hline Cortinarius scaurus (Fr.) Fr. & 翅鳞丝膜菌 & $\begin{array}{l}\text { Cystolepiota squamulosa (T. Bau \& Yu Li) } \\
\text { Zhu L. Yang }\end{array}$ & 红鳞囊小伞 \\
\hline
\end{tabular}


附录2 (续) Appendix 2 (continued)

拉丁学名
Dacryobolus phalloides Manjón, Hjortstam \&
G. Moreno

Dacryobolus sudans (Alb. \& Schwein.) Fr.

Dacryopinax fissus G. W. Martin

Daldinia caldariorum Henn.

Daldinia carpinicola Lar. N. Vassiljeva \& M. Stadler

Daldinia gelatinoides Lar. N. Vassiljeva

Datronia decipiens (Bres.) Ryvarden

Datronia glabra Ryvarden

Datronia perstrata (Corner) T. Hatt. \& Sotome

Datronia sepiicolor (Corner) T. Hatt. \& Sotome

Deconica phillipsii (Berk. \& Broome) Noordel.

Deconica phyllogena (Sacc.) Noordel.

Deflexula ulmi (Peck) Corner

Dendrocorticium polygonioides (P. Karst.)

M. J. Larsen \& Gilb.

Dendrothele alliacea (Quél.) P. A. Lemke

\begin{tabular}{l||} 
汉语学名 \\
鬼笔状耳壳菌
\end{tabular}

\begin{tabular}{|c|c|}
\hline 拉丁学名 & 汉语学名 \\
\hline Echinoderma pseudoasperulum (Knudsen) Bon & 假褐鳞鳞环柄菇 \\
\hline Efibula ginnsii (Sheng H. Wu) Zmitr. \& Spirin & 吉恩无锁革菌 \\
\hline Efibula lutea Sheng H. Wu & 土黄无锁革菌 \\
\hline Efibula subodontoidea (Sheng H. Wu) Zmitr. \& Spirin & 亚齿状无锁革菌 \\
\hline Efibulobasidium albescens (Sacc. \& Malbr.) K. Wells & 白无锁担耳 \\
\hline Eichleriella shearii (Burt) Spirin \& Malysheva & 席氏盘革耳 \\
\hline Entoloma aeruginosum Hiroë & 铜绿粉褶暵 \\
\hline Entoloma araneosum (Quél.) M. M. Moser & 蛛丝粉褶英 \\
\hline Entoloma concavosericeum Corner \& E. Horak & 绢状凹陷粉褶蕾 \\
\hline Entoloma corvinum (Kühner) Noordel. & 乌黑粉褶䒼 \\
\hline Entoloma decolorans E. Horak & 脱色粉褶䒼 \\
\hline Entoloma dinghuense T. H. Li \& Chuan H. Li & 鼎湖粉褶萆 \\
\hline Entoloma dysthales (Peck) Sacc. & 铁刀粉褶葷 \\
\hline Entoloma flavidum (Massee) Corner \& E. Horak & 黄粉褶草 \\
\hline Entoloma gasteromycetoides Co-David \& Noordel. & 腹粉褶蕾 \\
\hline
\end{tabular}

Dendrothele commixta (Höhn. \& Litsch.) J. Erikss. \& Ryvarden

Dentipellicula leptodon (Mont.) Y. C. Dai \&

L. W. Zhou

苏丹耳壳菌

裂纹假花耳

狭球轮层炭壳

我耳杵轮层炭壳

胶质轮层炭壳

迷惑异薄孔菌

光异薄孔菌

俯卧异薄孔菌

淡黑异薄孔菌

菲氏黄囊菇

叶生黄囊菇

榆龙爪菌

多角树状革菌

洋葱味树状皮 革菌

Dentocorticium ussuricum (Parmasto) M. J. Larsen \& Gilb.

Dermea acerina (Peck) Rehm

Dermea ariae (Pers.) Tul. \& C. Tul. ex P. Karst.

Descolea alba (Klotzsch) Kuhar, Nouhra \& M. E. Sm.

Descolea macrospora (G. Cunn.) Kuhar, Nouhra \& M. E. Sm.

Descolea pretiosa E. Horak

Deviodontia pilaecystidiata (S. Lundell) Hjortstam \& Ryvarden

Dextrinodontia molliuscula Hjortstam \& Ryvarden

Dichomitus sinuolatus H. S. Yuan

Dichostereum boreale (Pouzar) Ginns \&

M. N. L. Lefebvre

Dictyonema irrigatum (Berk. \& M. A. Curtis) Lücking

Dictyophora speciosa Meyen

Discina ancilis (Pers.) Sacc.

Discina caroliniana (Bosc) Eckblad

Ditiola abieticola D. A. Reid

Duportella kuehneroides Boidin, Lanq. \& Gilles

Duportella miranda Boidin, Lanq. \& Gilles

Duportella tristiculoides Sheng H. Wu \& Z. C. Chen

Echinoderma carinii (Bres.) Bon

Echinoderma echinaceum (J. E. Lange) Bon

Echinoderma jacobi (Vellinga \& Knudsen) Gminder
混合树状皮革菌

薄膜齿菌

乌苏里齿伏革菌 槭生皮盘菌 阿里皮盘菌

白圆头伞

大狍圆头伞

普雷蒂圆头伞

细囊弯齿革菌

软糊精齿菌

波状叉丝孔菌

北方双叉韧革菌

灌溉云片衣

美竹䔉

平盘菌

肉色平盘菌

冷杉韧钉耳

类克氏硬孔革菌

黄纱硬孔革菌

类毛硬孔革菌

棕色鳞环柄菇

刺鳞鳞环柄菇

雉鳞鳞环柄菇
Entoloma gnaphalodes (Berk. \& Broome) E. Horak

Entoloma griseocyaneum (Fr.) P. Kumm.

鼠曲草粉褶萆

Entoloma hesleri Morgan-Jones

灰蓝粉褶草

赫氏粉褶蔁

Entoloma infuscatum Hesler

Entoloma jubatum (Fr.) P. Karst.

带褐粉褶草

鬃粉褶蔁

Entoloma juncinum (Kühner \& Romagn.) Noordel. 接合粉褶䓦

Entoloma kauffmanii Malloch

考夫曼粉褶萆

Entoloma leptoniisporum (Richon) J. M. Vidal \&

P.-A. Moreau

Entoloma longistriatum (Peck) Noordel.

薄狍粉褶蔁

Entoloma mammulatum Hesler

长条纹粉褶蔁

Entoloma mastoideum T. H. Li \& Xiao L. He

凸粉褶蔁

乳头状粉褶蔁

Entoloma neglectum (Lasch) Arnolds

疏忽粉褶草

Entoloma nigrosquamosum Hesler

黑鳞粉褶萆

Entoloma parasiticum (Quél.) Kreisel

寄生粉褶草

Entoloma parkensis (Fr.) Noordel.

公园粉褶草

Entoloma proprium E. Horak

固有粉褶草

Entoloma rusticoides (Gillet) Noordel.

锈红粉褶萆

Entoloma sphagnorum (Romagn. \& J. Favre) Bon \&

泥炭藓粉褶蔁 Courtec.

Entoloma strigosissimum (Rea) Noordel.

硬毛粉褶萆

Epithele macarangae Boidin \& Lanq.

瘦弱上皮孔菌

Epithele nikau G. Cunn.

尼考上皮孔菌

Evernia prunastri (L.) Ach.

普氏扁枝衣

Exidiopsis alliciens (Berk. \& Cooke) K. Wells 
附录2 (续) Appendix 2 (continued)

\begin{tabular}{|c|c|c|c|}
\hline 拉丁学名 & 汉语学名 & 拉丁学名 & 汉语学名 \\
\hline Exidiopsis galzinii (Bres.) Killerm. & 蜡拟黑耳 & |Geastrum quadrifidum DC. ex Pers. & 四裂地星 \\
\hline Exidiopsis pallida K. Wells \& Raitv. & 苍白拟黑耳 & |Geopora tenuis (Fuckel) T. Schumach. & 薄地孔菌 \\
\hline Fibricium rude (P. Karst.) Jülich & 皱索革菌 & Gerronema reclinis (Fr.) Clémençon & 拱垂老伞 \\
\hline Fistulinella nana (Massee) E. Horak & 微小牛排菌 & Globulicium hiemale (Laurila) Hjortstam & 冬生球瑚菌 \\
\hline Flammula picea Velen. & 云杉火菇 & Gloeocystidiellum aspellum Hjortstam & 阿地胶囊伏革菌 \\
\hline Flammulaster ferrugineus Maire ex Watling & 锈色暗皮伞 & Gloeocystidiellum compactum Sheng H. Wu & 致密胶囊伏革菌 \\
\hline Flammulaster muricatus (Fr.) Watling & 粗糙暗皮伞 & Gloeocystidiellum convolvens (P. Karst.) Donk & 共托胶囊伏革菌 \\
\hline Flammulaster siparius (Fr.) Watling & 锈褐暗皮伞 & Gloeocystidiellum formosanum Sheng $\mathrm{H}$. Wu & 台湾胶囊伏革菌 \\
\hline Flammulaster wieslandri (Fr.) M. M. Moser & 韦氏暗皮伞 & Gloeocystidiellum heimii Boidin & 黑姆胶囊伏革菌 \\
\hline Flaviporus hydrophilus (Berk. \& M. A. Curtis) Ginns & 喜湿黄孔菌 & Gloeocystidiellum laxum Sheng H. Wu & 松胶囊伏革菌 \\
\hline Flaviporus stramineus (Bres.) Ginns & 麦黄黄孔菌 & Gloeocystidiellum moniliforme Sheng $\mathrm{H}$. Wu & 念珠胶囊伏革菌 \\
\hline Fomes fasciatus (Sw.) Cooke & 簇生层孔菌 & Gloeocystidiellum purpureum Sheng $\mathrm{H}$. Wu & 紫胶囊伏革菌 \\
\hline Fomes nigrolaccatus (Cooke) Sacc. & 黑漆层孔菌 & Gloeocystidiellum tabacinum Sheng $\mathrm{H}$. Wu & $\begin{array}{l}\text { 烟草色胶囊伏 } \\
\text { 革菌 }\end{array}$ \\
\hline Fomitella supina (Sw.) Murrill & 仰卧小层孔菌 & $\begin{array}{l}\text { Gloeodontia subasperispora (Litsch.) E. Larss. \& } \\
\text { K. H. Larss. }\end{array}$ & 粘狍粘齿菌 \\
\hline Fomitiporella caviphila L. W. Zhou & 空小层卧孔菌 & $\begin{array}{l}\text { Gloeohypochnicium analogum (Bourdot \& Galzin) } \\
\text { Hjortstam }\end{array}$ & 相似粘纹菌 \\
\hline Fomitiporia pentaphylacis L. W. Zhou & 五角层卧孔菌 & Gloeomyces ginnsii Sheng H. Wu & 吉恩粘革菌 \\
\hline Fomitiporia tenuitubus L. W. Zhou & 薄管层卧孔菌 & Gloeomyces graminicola Sheng $\mathrm{H}$. Wu & 禾生粘革菌 \\
\hline Fomitopsis persoonii (Fr.) Imaz. & 珀松拟层孔菌 & Gloeomyces moniliformis (N. Maek.) Sheng H. Wu & 念珠粘革菌 \\
\hline Frantisekia abieticola H. S. Yuan & 冷杉弗兰孔菌 & Gloiothele citrina (Pers.) Ginns \& G. W. Freeman & 柠檬黄黏革菌 \\
\hline $\begin{array}{l}\text { Fulvifomes membranaceus (J. E. Wright \& Blumenf.) } \\
\text { Baltazar \& Gibertoni }\end{array}$ & 膜黄褐层孔菌 & Gloiothele citrinoidea Sheng $\mathrm{H}$. Wu & 近柠檬黄黏革菌 \\
\hline $\begin{array}{l}\text { Fuscoporia longisetulosa (Bondartseva \& S. Herrera) } \\
\text { Bondartseva \& S. Herrera }\end{array}$ & 长刺褐孔菌 & Gloiothele globosa Sheng H. Wu & 球黏革菌 \\
\hline Galera campanulata Massee & 钟形帽伞 & Grammothelopsis subtropica B. K. Cui \& C. L. Zhao & 亚热带拟线齿菌 \\
\hline Galerella plicatella (Peck) Singer & 褶纹小帽伞 & Grandinia muscicola (Pers.) Bres. ex Bourdot \& Galzin & n苔生格兰齿菌 \\
\hline Galerina atkinsoniana A. H. Sm. & 爱其逊盔孢伞 & Guepinia buccina Sacc. & 贝状桂花耳 \\
\hline Galerina badipes (Pers.) Kühner & 褐盔狍伞 & Guepiniopsis alpina (Earle) Brasf. & 高山胶盘耳 \\
\hline Galerina cerina A. H. Sm. \& Singer & 蜡黄盔孢伞 & |Guepiniopsis estonica (Raitv.) M. Dueñas & 爱沙尼亚胶盘耳 \\
\hline Galerina evelata (Singer) A. H. Sm. \& Singer & 无菌幕盛孢伞 & Gymnopilus hybridus (Gillet) Maire & 杂裸伞 \\
\hline Galerina salicicola P. D. Orton & 柳盔孢伞 & $\begin{array}{l}\text { Gymnopus castaneus M. Villarreal, Heykoop \& } \\
\text { Esteve-Rav. }\end{array}$ & 栗色裸柄伞 \\
\hline Galerina sphagnorum (Pers.) Kühner & 泥炭藓盔孢伞 & \begin{tabular}{|l} 
Gymnopus fuscotramus Mešić, Tkalčec \& \\
Chun Y. Deng
\end{tabular} & 暗裸柄伞 \\
\hline Galerina stylifera (G. F. Atk.) A. H. Sm. \& Singer & 柱盔孢伞 & Gymnopus impudicus (Fr.) Antonín, Halling \& Noordel. & . 厌裸柄伞 \\
\hline Galerina triscopa (Fr.) Kühner & 三域盔狍伞 & $\begin{array}{l}\text { Gyrophanopsis polonensis (Bres.) Stalpers \& } \\
\text { P. K. Buchanan }\end{array}$ & 波兰拟圆炷菌 \\
\hline Galerina uncialis (Britzelm.) Kühner & 钩形盔孢伞 & Hebeloma album Peck & 白粘滑菇 \\
\hline Galiella japonica (Yasuda) Y. Otani & 日本盖尔盘菌 & Hebeloma birrus (Fr.) Gillet & 活力粘滑菇 \\
\hline Galiella sinensis J. Z. Cao & 中国盖尔盘菌 & Hebeloma ingratum Bruchet & 短柄粘滑菇 \\
\hline Ganoderma adspersum (Schulzer) Donk & 广布灵芝 & Hebeloma pusillum J. E. Lange & 小粘滑菇 \\
\hline $\begin{array}{l}\text { Gastroleccinum scabrosum (Mazzer \& A. H. Sm.) } \\
\text { Thiers }\end{array}$ & $\begin{array}{l}\text { 粗糙腹疮柄牛 } \\
\text { 肝菌 }\end{array}$ & Hebeloma vaccinum Romagn. & 暗褐粘滑菇 \\
\hline Gautieria xinjiangensis T. Bau & 新疆高腹菌 & Helvella exarata Gillet & 斑纹马鞍菌 \\
\hline Geastrum lageniforme Vittad. & 葫芦形地星 & $\begin{array}{l}\text { Hemileccinum indecorum (Massee) G. Wu \& } \\
\text { Zhu L. Yang }\end{array}$ & $\begin{array}{l}\text { 无饰半痰柄牛 } \\
\text { 肝菌 }\end{array}$ \\
\hline
\end{tabular}


附录2 (续) Appendix 2 (continued)

\begin{tabular}{|c|c|c|c|}
\hline 拉丁学名 & 汉语学名 & 拉丁学名 & 汉语学名 \\
\hline Hemimycena cucullata (Pers.) Singer & 帽形半小菇 & Hygrocybe quieta (Kühner) Singer & 安静湿伞 \\
\hline Hemimycena hirsuta (Tode) Singer & 硬毛半小菇 & Hygrocybe subceracea Murrill & 蜡湿伞 \\
\hline Hemimycena pseudolactea (Kühner) Singer & 假白半小菇 & Hygrocybe swanetica Singer & 丝旺尼湿伞 \\
\hline Hemimycena rickenii (A. H. Sm.) Singer & 里肯半小菇 & Hygrophorus albidus P. Karst. & 白蜡伞 \\
\hline Hemipholiota heteroclita (Fr.) Bon & 异果半鳞伞 & Hygrophorus lindtneri M. M. Moser & 林特纳蜡伞 \\
\hline Henningsomyces leptus Y. L. Wei \& Y. C. Dai & 小哈宁管菌 & Hygrophorus miniaceus Beck & 亮红蜡伞 \\
\hline Heterobasidion abietinum Niemelä \& Korhonen & 冷杉异担子菌 & Hygrophorus pictus Berk. \& M. A. Curtis & 着色蜡伞 \\
\hline $\begin{array}{l}\text { Heterochaete spinulosa (Berk. \& M. A. Curtis) } \\
\text { D. A. Reid }\end{array}$ & 细刺刺皮菌 & Hygrophorus pustulatus (Pers.) Fr. & 疱突蜡伞 \\
\hline $\begin{array}{l}\text { Heterorepetobasidium ellipsoideum Oberw. \& } \\
\text { Chee J. Chen }\end{array}$ & 椭圆异重担菌 & Hygrophorus queletii Bres. & 凯莱蜡伞 \\
\hline $\begin{array}{l}\text { Heterorepetobasidium subglobosum Chee J. Chen \& } \\
\text { Oberw. }\end{array}$ & 亚球孢异重担菌 & Hymenochaete acerosa S. H. He \& Hai J. Li & 槭生刺革菌 \\
\hline $\begin{array}{l}\text { Hjortstamia novae-granata (A. L. Welden) Hjortstam \& } \\
\text { Ryvarden }\end{array}$ & 晶约氏革菌 & Hymenochaete borealis Burt & 北方刺革菌 \\
\hline Hohenbuehelia approximans (Peck) Singer & 紧靠亚侧耳 & Hymenochaete odontoides S. H. He \& Y. C. Dai & 齿状刺革菌 \\
\hline Hohenbuehelia concurrens (Drechsler) Thorn & 并发亚侧耳 & Hymenochaete quercicola S. H. He \& Hai J. Li & 栎生刺革菌 \\
\hline Hohenbuehelia cyphelliformis (Berk.) O. K. Mill. & 杯点亚侧耳 & Hymenochaete semistupposa Petch & 半卷毛刺革菌 \\
\hline Hohenbuehelia haptoclada (Drechsler) Thorn & 硬亚侧耳 & Hymenochaete tenuis Peck & 纤细刺革菌 \\
\hline Hohenbuehelia horakii Courtec. & 霍氏亚侧耳 & Hymenochaete ustulata G. A. Escobar & 枯焦刺革菌 \\
\hline Hohenbuehelia leiospora (Drechsler) Thorn & 平狍亚侧耳 & $\begin{array}{l}\text { Hymenochaetopsis latesetosa (S. H. He \& Hai J. Li) } \\
\text { S. H. He \& Jiao Yang }\end{array}$ & 砖红拟刺革菌 \\
\hline Hohenbuehelia leptospora (Drechsler) Thorn & 薄狍亚侧耳 & Hymenopellis colensoi (Dörfelt) R. H. Petersen & 科伦索长根菇 \\
\hline Hohenbuehelia longipes (Boud.) M. M. Moser & 长柄亚侧耳 & $\begin{array}{l}\text { Hymenopellis vinocontusa (R. H. Petersen \& Nagas.) } \\
\text { R. H. Petersen }\end{array}$ & 酒红长根菇 \\
\hline Hohenbuehelia mastrucata (Fr.) Singer & 灰黄鳞亚侧耳 & Hyphoderma albicans (Pers.) Nakasone & 白丝皮菌 \\
\hline Hohenbuehelia myxotricha (Lév.) Singer & 粘毛亚侧耳 & Hyphoderma amoenum (Burt) Donk & 怡丝皮菌 \\
\hline Hohenbuehelia pinacearum Thorn & 松亚侧耳 & Hyphoderma ayresii (Berk. ex Cooke) Boidin \& Gilles & 埃尔斯丝皮菌 \\
\hline $\begin{array}{l}\text { Hohenbuehelia subreniformis (Thorn \& G. L. Barron) } \\
\text { Thorn }\end{array}$ & 亚肾形亚侧耳 & Hyphoderma clavatum Sheng H. Wu & 棒丝皮菌 \\
\hline $\begin{array}{l}\text { Hohenbuehelia tremula (Schaeff.) Thorn \& } \\
\text { G. L. Barron }\end{array}$ & 胶质亚侧耳 & Hyphoderma cremeum Sheng H. Wu & 奶油丝皮菌 \\
\hline $\begin{array}{l}\text { Hohenbuehelia tripolitania (Giuma \& R. C. Cooke) } \\
\text { Thorn }\end{array}$ & 的黎亚侧耳 & Hyphoderma definitum (H. S. Jacks.) Donk & 明确丝皮菌 \\
\hline Hohenbuehelia tylospora (Drechsler) Thorn & 黑灰孢亚侧耳 & Hyphoderma densum Sheng H. Wu & 密丝皮菌 \\
\hline Homophron cernuum (Vahl) Örstadius \& E. Larss. & 俯垂类脆柄菇 & Hyphoderma hjortstamii Sheng H. Wu & 霍氏丝皮菌 \\
\hline Hydnellum caeruleum (Hornem.) P. Karst. & 蓝亚齿菌 & $\begin{array}{l}\text { Hyphoderma malenconii (Manjón \& G. Moreno) } \\
\text { Manjón, G. Moreno \& Hjortstam }\end{array}$ & 玛氏丝皮菌 \\
\hline Hydnellum regium K. A. Harrison & 桃红亚齿菌 & $\begin{array}{l}\text { Hyphoderma moniliforme (P. H. B. Talbot) Manjón, } \\
\text { G. Moreno \& Hjortstam }\end{array}$ & 念珠丝皮菌 \\
\hline Hydnellum scrobiculatum (Fr.) P. Karst. & 蜂窝亚齿菌 & Hyphoderma nemorale K. H. Larss. & 林生丝皮菌 \\
\hline Hydnellum spongiosipes (Peck) Pouzar & 海绵亚齿菌 & $\begin{array}{l}\text { Hyphoderma occidentale (D. P. Rogers) Boidin \& } \\
\text { Gilles }\end{array}$ & 西方丝皮菌 \\
\hline Hydnocristella himantia (Schwein.) R. H. Petersen & 扇索状刺顶菌 & Hyphoderma rimulosum Sheng $\mathrm{H} . \mathrm{Wu}$ & 裂纹丝皮菌 \\
\hline $\begin{array}{l}\text { Hydnotrya laojunshanensis Lin Li, Y. C. Zhao, } \\
\text { X. Lei Zhang, Shu H. Li \& D. Q. Zhou }\end{array}$ & 老君山腔块菌 & Hyphoderma subclavatum Sheng H. Wu & 亚棒状丝皮菌 \\
\hline Hydropus fuliginarius (Batsch) Singer & 烟煤湿柄伞 & Hyphoderma subsetigerum Sheng H. Wu & 亚刚毛丝皮菌 \\
\hline Hygrocybe cruenta (Hongo) Hongo & 血色湿伞 & Hyphoderma tenue (Pat.) Donk & 纤细丝皮菌 \\
\hline Hygrocybe cystidiata Arnolds & 囊湿伞 & Hyphoderma typhicola (Burt) Donk & 香蒲丝皮菌 \\
\hline Hygrocybe insipida (J. E. Lange) M. M. Moser & 淡湿伞 & Hyphoderma variolosum Boidin, Lanq. \& Gilles & 颗粒丝皮菌 \\
\hline
\end{tabular}


附录2 (续) Appendix 2 (continued)

\begin{tabular}{|c|c|c|c|}
\hline 拉丁学名 & 汉语学名 & 拉丁学名 & 汉语学名 \\
\hline $\begin{array}{l}\text { Hyphodontia cineracea (Bourdot \& Galzin) J. Erikss. \& } \\
\text { Hjortstam }\end{array}$ & 灰产丝齿菌 & Hypoxylon musceum J. D. Rogers & 粘炭团菌 \\
\hline Hyphodontia dimorpha Sang H. Lin \& Z. C. Chen & 二型产丝齿菌 & Hypoxylon parksianum Y. M. Ju \& J. D. Rogers & 木槿炭团菌 \\
\hline Hyphodontia microspora J. Erikss. \& Hjortstam & 小孢产丝齿菌 & Hypoxylon retpela Van der Gucht \& Van der Veken & 粉红炭团菌 \\
\hline Hyphodontia mollis Sheng H. Wu & 软产丝齿菌 & Hypoxylon subgilvum Berk. \& Broome & 亚铜色炭团菌 \\
\hline Hyphodontia propinqua Hjortstam & 邻产丝齿菌 & Hypoxylon trugodes Berk. \& Broome & 松杉炭团菌 \\
\hline $\begin{array}{l}\text { Hyphodontia septocystidiata H. X. Xiong, Y. C. Dai \& } \\
\text { Sheng H. Wu }\end{array}$ & 隔囊产丝齿菌 & Hypoxylon ulmophilum Lar. N. Vassiljeva & 榆炭团菌 \\
\hline $\begin{array}{l}\text { Hyphodontia subpallidula H. X. Xiong, Y. C. Dai \& } \\
\text { Sheng H. Wu }\end{array}$ & 亚淡色产丝齿菌 & |Hypoxylon vogesiacum (Pers. ex Curr.) Sacc. & 卷边炭团菌 \\
\hline Hyphodontia subspathulata (H. Furuk.) N. Maek. & 亚匙形产丝齿菌 & $\begin{array}{l}\text { Hypoxylon yunnanense Hai X. Ma, } \\
\text { Lar. N. Vassiljeva \& Yu Li }\end{array}$ & 云南炭团菌 \\
\hline Hypholoma acutum (Sacc.) E. Horak & 尖垂幕菇 & $\begin{array}{l}\text { Imleria obscurebrunnea (Hongo) Xue T. Zhu \& } \\
\text { Zhu L. Yang }\end{array}$ & 暗棕褐牛肝菌 \\
\hline Hypholoma elatum Massee & 弹丝垂幕菇 & $\begin{array}{l}\text { Imperator rhodopurpureus (Smotl.) Assyov, Bellanger, } \\
\text { Bertéa, Courtec., Koller, Loizides, G. Marques, J. A. } \\
\text { Muñoz, Oppicelli, D. Puddu, F. Rich. \& P.-A. Moreau }\end{array}$ & , 朱孔皇牛肝菌 \\
\hline Hypholoma elongatipes C. S. Parker & 长柄垂幕菇 & Inflatostereum glabrum (Pat.) D. A. Reid & 光膨大韧革菌 \\
\hline Hypholoma elongatum (Pers.) Ricken & 长垂幕菇 & Inocybe acuta Boud. & 尖丝盖伞 \\
\hline Hypochnicium erikssonii Hallenb. \& Hjortstam & 爱立逊纹革菌 & Inocybe albomarginata Velen. & 银边丝盖伞 \\
\hline Hypocrea aurantia Henn. & 橙黄肉座菌 & Inocybe amethystina Kuyper & 紫晶丝盖伞 \\
\hline Hypocrea brunneolutea Yoshim. Doi & 褐肉座菌 & Inocybe brunneotomentosa Huijsman & 褐茸丝盖伞 \\
\hline Hypocrea flavovirens Berk. & 黄绿肉座菌 & Inocybe chalcodoxantha Grund \& D. E. Stuntz & 铜黄丝盖伞 \\
\hline Hypocrea megalosulphurea Yoshim. Doi & 大硫色肉座菌 & Inocybe fraudans (Britzelm.) Sacc. & 长柄丝盖伞 \\
\hline Hypocrea muroiana I. Hino \& Katum. & 竹生肉座菌 & Inocybe fuscidula Velen. & 黑丝盖伞 \\
\hline Hypocrea nigricans (S. Imai) Yoshim. Doi & 黑肉座菌 & Inocybe fuscodisca (Peck) Massee & 暗盘丝盖伞 \\
\hline Hypocrea pachybasioides Yoshim. Doi & 厚担肉座菌 & Inocybe glabripes Ricken & 光柄丝盖伞 \\
\hline Hypoxylon brevisporum Y. M. Ju \& J. D. Rogers & 短狍炭团菌 & Inocybe langei R. Heim & 兰格丝盖伞 \\
\hline $\begin{array}{l}\text { Hypoxylon cercidicola (Berk. \& M. A. Curtis ex Peck) } \\
\text { Y. M. Ju \& J. D. Rogers }\end{array}$ & 废退炭团菌 & Inocybe leptocystis G. F. Atk. & 薄囊丝盖伞 \\
\hline Hypoxylon cinnabarinum (Henn.) Henn. & 朱红炭团菌 & Inocybe margaritispora (Berk.) Sacc. & 珍珠丝盖伞 \\
\hline Hypoxylon crocopeplum Berk. \& M. A. Curtis & 杏黄炭团菌 & Inocybe mixtilis (Britzelm.) Sacc. & 混杂丝盖伞 \\
\hline Hypoxylon dearnessii Y. M. Ju \& J. D. Rogers & 戴氏炭团菌 & Inocybe miyiensis T. Bau \& Y. G. Fan & 米易丝盖伞 \\
\hline $\begin{array}{l}\text { Hypoxylon dengii Hai X. Ma, Lar. N. Vassiljeva \& } \\
\text { Yu Li }\end{array}$ & 邓氏炭团菌 & Inocybe muricellata Bres. & 䊁柄丝盖伞 \\
\hline Hypoxylon duranii J. D. Rogers & 杜兰炭团菌 & Inocybe niigatensis Hongo & 新泻丝盖伞 \\
\hline Hypoxylon fendleri Berk. ex Cooke & 芬德勒炭团菌 & Inocybe nobilis (R. Heim) Alessio & 高贵丝盖伞 \\
\hline Hypoxylon fraxinophilum Pouzar & 白蜡炭团菌 & Inocybe obscurobadia (J. Favre) Grund \& D. E. Stuntz & 遮蔽丝盖伞 \\
\hline Hypoxylon fuscopurpureum (Schwein.) M. A. Curtis & 暗紫炭团菌 & Inocybe ochroalba Bruyl. & 黄白丝盖伞 \\
\hline Hypoxylon griseocinctum (Starbäck) P. M. D. Martin & 灰炭团菌 & Inocybe perlata (Cooke) Sacc. & 光泽丝盖伞 \\
\hline $\begin{array}{l}\text { Hypoxylon hubeiense Hai X. Ma, Lar. N. Vassiljeva \& } \\
\text { Yu Li }\end{array}$ & 湖北炭团菌 & Inocybe posterula (Britzelm.) Sacc. & 后腔丝盖伞 \\
\hline Hypoxylon investiens (Schwein.) M. A. Curtis & 坚硬炭团菌 & Inocybe sindonia (Fr.) P. Karst. & 毒丝盖伞 \\
\hline Hypoxylon karii Y. M. Ju \& J. D. Rogers & 卡尔炭团菌 & Inocybe sororia Kauffman & 成堆丝盖伞 \\
\hline Hypoxylon kretzschmarioides Y. M. Ju \& J. D. Rogers & 克雷炭团菌 & Inocybe splendens R. Heim & 光亮丝盖伞 \\
\hline Hypoxylon laschii Nitschke & 拉什炭团菌 & Inocybe subcarpta Kühner \& Boursier & 亚果丝盖伞 \\
\hline Hypoxylon lienhwacheense Y. M. Ju \& J. D. Rogers & 台湾炭团菌 & Inocybe tenebrosa Quél. & 阴暗丝盖伞 \\
\hline Hypoxylon lividicolor Y. M. Ju \& J. D. Rogers & 铅色炭团菌 & Inocybe whitei (Berk. \& Broome) Sacc. & 怀特丝盖伞 \\
\hline Hypoxylon macrosporum P. Karst. & 巨孢炭团菌 & Inonotus acutus B. K. Cui \& Y. C. Dai & 尖纤孔菌 \\
\hline
\end{tabular}


附录2 (续) Appendix 2 (continued)

\begin{tabular}{|c|c|c|c|}
\hline 拉丁学名 & 汉语学名 & 拉丁学名 & 汉语学名 \\
\hline Inonotus canariicola Y. C. Dai & 贾纳尔纤孔菌 & Leccinum leucophaeum (Pers.) Bon & 白疮柄牛肝菌 \\
\hline Inonotus chilanshanus T. T. Chang \& W. N. Chou & 赤兰山纤孔菌 & Lecidea atrobrunnea (DC.) Schaer. & 黑棕网衣 \\
\hline Inonotus indurescens Y. C. Dai & 变硬纤孔菌 & Lecidea hypocrita A. Massal. & 伪网衣 \\
\hline Inonotus magnisetus Y. C. Dai & 巨毛纤孔菌 & Lentinellus micheneri (Berk. \& M. A. Curtis) Pegler & 米切纳螺壳菌 \\
\hline Inonotus splitgerberi (Mont.) Ryvarden & 斯氏纤孔菌 & Lentinellus tridentinus (Sacc. \& P. Syd.) Singer & 三齿螺壳菌 \\
\hline Ionomidotis irregularis (Schwein.) E. J. Durand & 畸聚盘菌 & Lentinula boryana (Berk. \& Mont.) Pegler & 卷木菇 \\
\hline Ioplaca pindarensis (Räsänen) Poelt \& Hinter. & 粉胎座衣 & Lentinula novae-zelandiae (G. Stev.) Pegler & 新西兰木菇 \\
\hline Jaapia ochroleuca (Bres.) Nannf. \& J. Erikss. & 黄白夏氏伏革菌 & Lentinus levis (Berk. \& M. A. Curtis) Murrill & 光滑㓞伞 \\
\hline $\begin{array}{l}\text { Jacksonomyces pseudocretaceus Sheng H. Wu \& } \\
\text { Z. C. Chen }\end{array}$ & 假石灰杰氏孔菌 & Lentinus odorus Quél. & 芳香㓞伞 \\
\hline Jansia boninensis Lloyd & 博尼疮盖鬼笔 & Lentinus substrictus (Bolton) Zmitr. \& Kovalenko & 亚致密㓞伞 \\
\hline Jansia elegans Penz. & 雅致病盖鬼笔 & Lepiota albissima (Murrill) Murrill & 变环柄菇 \\
\hline Junghuhnia flabellata H. S. Yuan \& Y. C. Dai & 扇形容氏孔菌 & Lepiota augustana (Britzelm.) Sacc. & 大紫环柄菇 \\
\hline Kavinia alboviridis (Morgan) Gilb. \& Budington & 变绿凯文菌 & Lepiota boudieri Bres. & 布迪耶环柄菇 \\
\hline Kneiffiella serpentiformis (Langer) Riebesehl \& Langer & 蛇皮奈氏齿菌 & Lepiota echinella Quél. \& G. E. Bernard & 缘毛环柄菇 \\
\hline $\begin{array}{l}\text { Kneiffiella sinensis (H. X. Xiong, Y. C. Dai \& } \\
\text { Sheng H. Wu) Riebesehl \& Langer }\end{array}$ & 中国奈氏齿菌 & Lepiota grangei (Eyre) Kühner & 格兰环柄菇 \\
\hline Laccaria acanthospora A. W. Wilson \& G. M. Muell. & 棘狍蜡蘑 & Lepiota neophana Morgan & 新环柄菇 \\
\hline Laccaria bullipellis A. W. Wilson \& G. M. Muell. & 泡状蜡蘑 & Lepiota otsuensis (Hongo) Hongo & 褐紫鳞环柄菇 \\
\hline Laccaria negrimarginata A. W. Wilson \& G. M. Muell. & 棕黑蜡蘑 & Lepiota praetervisa Hongo & 瞭望环柄菇 \\
\hline Laccaria ohiensis (Mont.) Singer & 俄亥俄蜡蘑 & Lepiota pseudofelina J. E. Lange & 假细环柄菇 \\
\hline Laccaria salmonicolor A. W. Wilson \& G. M. Muell. & 鲑色蜡蘑 & Lepiota rhachoderma (Berk. \& Broome) Sacc. & 脊环柄菇 \\
\hline Lactarius albocarneus Britzelm. & 白肉色乳菇 & Lepiota rufipes Morgan & 暗红环柄菇 \\
\hline Lactarius areolatus Hesler \& A. H. Sm. & 网隙乳菇 & Lepiota subalba Kühner ex P. D. Orton & 亚白环柄菇 \\
\hline Lactarius aurantiaco-ochraceus Lj. N. Vassiljeva & 橙赦乳菇 & Lepiota thrombophora (Berk. \& Broome) Sacc. & 栓狍环柄菇 \\
\hline Lactarius castanopsidis Hongo & 栲乳菇 & Lepiota tomentella J. E. Lange & 鸾毛环柄菇 \\
\hline Lactarius delicatus Burl. & 柔美乳菇 & Lepiota virescens (Speg.) Morgan & 变绿环柄菇 \\
\hline Lactarius fulvissimus Romagn. & 褐乳菇 & Lepiota wasseri Bon & 瓦塞尔环柄菇 \\
\hline Lactarius grandisporus Lj. N. Vassiljeva & 大孢乳菇 & Lepiota xanthophylla P. D. Orton & 黄叶环柄菇 \\
\hline Lactarius nigroviolascens G. F. Atk. & 黑紫乳菇 & Leptogium caesium (Ach.) Vain. & 蓝猫耳衣 \\
\hline Lactarius obscuratus (Lasch) Fr. & 暗乳菇 & $\begin{array}{l}\text { Leucoagaricus atrosquamulosus (Hongo) Z. W. Ge \& } \\
\text { Zhu L. Yang }\end{array}$ & 黑鳞白环蘑 \\
\hline Lactarius sakamotoi S. Imai & 坂本乳菇 & Leucoagaricus brunneocingulatus (P. D. Orton) Bon & 肉褐鳞白环蘑 \\
\hline Lactarius vinaceorufescens A. H. Sm. & 酒红乳菇 & Leucoagaricus georginae (W. G. Sm.) Candusso & 若尔然白环蘑 \\
\hline Langermannia maxima (Schaeff.) Pázmány & 大马线菇 & $\begin{array}{l}\text { Leucoagaricus lateritiopurpureus (Lj. N. Vassiljeva) } \\
\text { E. F. Malysheva, Svetash. \& Bulakh }\end{array}$ & 砖红紫白环蘑 \\
\hline $\begin{array}{l}\text { Lanmaoa fragrans (Vittad.) Vizzini, Gelardi \& } \\
\text { Simonini }\end{array}$ & 芳香兰茂牛肝菌|| & Leucoagaricus meleagris (Gray) Singer & 西方平盖白环蘑 \\
\hline Laternea columnata Nees & 柱状拱门菌 & \begin{tabular}{|l} 
Leucoagaricus purpureoruber (Z. S. Bi, T. H. Li \& \\
G. Y. Zheng) Z. W. Ge \& Zhu L. Yang
\end{tabular} & 紫红白环蘑 \\
\hline $\begin{array}{l}\text { Lauriliella taxodii (Lentz \& H. H. McKay) S. H. He \& } \\
\text { Nakasone }\end{array}$ & 紫杉小劳里菌 & Leucoagaricus sericifer (Locq.) Vellinga & 小褐白环蘑 \\
\hline Lecanora sulphurescens Fée & 近硫色茶渍 & Leucocoprinus straminellus (Bagl.) Narducci \& Caroti & 麦黄白鬼伞 \\
\hline Leccinellum pseudoscabrum (Kallenb.) Mikšík & $\begin{array}{l}\text { 假糙小疮柄牛 } \\
\text { 肝菌 }\end{array}$ & Leucogaster nudus (Hazsl.) Hollós & 裸白腹菌 \\
\hline Leccinum barrowsii A. H. Sm., Thiers \& Watling & 巴氏疮柄牛肝菌|| & $\begin{array}{l}\text { Leucopaxillus nauseosodulcis (P. Karst.) Singer \& } \\
\text { A. H. Sm. }\end{array}$ & 臭味白桩菇 \\
\hline Leccinum flavostipitatum E. A. Dick \& Snell & 黄柄疮柄牛肝菌| & |Lignosus superpositus (Berk.) Lloyd. & 叠生木孔菌 \\
\hline
\end{tabular}


附录2 (续) Appendix 2 (continued)

\begin{tabular}{|c|c|c|c|}
\hline 拉丁学名 & 汉语学名 & 拉丁学名 & 汉语学名 \\
\hline Lycoperdon decipiens Durieu \& Mont. & 迷惑马勃 & Montagnea haussknechtii Rabenh. & 霍氏蒙塔假菇 \\
\hline Lycoperdon echinulatum Berk. \& Broome & 小刺马勃 & Morchella canina Leuba & 尖羊肚菌 \\
\hline Lycoperdon fucatum Lév. & 光滑白马勃 & \begin{tabular}{|l} 
Morchella deqinensis Shu H. Li, Y. C. Zhao, \\
H. M. Chai \& M. H. Zhong
\end{tabular} & 德清羊肚菌 \\
\hline Lycoperdon muscorum Morgan & 苔生马勃 & Morchella ovalis (Wallr.) Boud. & 卵形羊肚菌 \\
\hline Lycoperdon nigrescens Pers. & 变黑马勃 & Morchella tridentina Bres. & 三齿羊肚菌 \\
\hline Lycoperdon norvegicum Demoulin & 挪威马勃 & Mutatoderma heterocystidia (Burt) C. E. Gómez & 异囊丝皮革菌 \\
\hline Lycoperdon papillatum Schaeff. & 乳突马勃 & Mutatoderma populneum (Peck) C. E. Gómez & 杨丝皮革菌 \\
\hline Lycoperdon polycephalum Lloyd & 多头马勃 & Mycena amicta (Fr.) Quél. & 长柄小菇 \\
\hline $\begin{array}{l}\text { Lyomyces capitatocystidiatus (H. X. Xiong, Y. C. Dai \& } \\
\text { Sheng H. Wu) Riebesehl \& Langer }\end{array}$ & 头囊疏伏革菌 & Mycena californiensis (Berk. \& M. A. Curtis) Sacc. & 加州小菇 \\
\hline Lyomyces pruni (Lasch) Riebesehl \& Langer & 李生疏伏革菌 & Mycena citrinomarginata Gillet & 橘色凹小菇 \\
\hline $\begin{array}{l}\text { Lyomyces tenuissimus (Yurchenko \& Sheng H. Wu) } \\
\text { Riebesehl \& Langer }\end{array}$ & 薄疏伏革菌 & Mycena coracina Maas Geest. & 乌黑小菇 \\
\hline $\begin{array}{l}\text { Macrocybe titans (H. E. Bigelow \& Kimbr.) Pegler, } \\
\text { Lodge \& Nakasone }\end{array}$ & 巨型大口蘑 & $\begin{array}{l}\text { Mycena dryopteriphila Lj. N. Vassiljeva \& } \\
\text { M. M. Nazarova }\end{array}$ & 栋小菇 \\
\hline Marasmiellus chamaecyparidis (Hongo) Hongo & 矮生微皮伞 & Mycena egregia Maas Geest. & 异常小菇 \\
\hline Marasmiellus phaeophyllus (Kühner) Singer & 褐褶微皮伞 & Mycena erubescens Höhn. & 变红小菇 \\
\hline Marasmiellus tricolor (Alb. \& Schwein.) Singer & 三色微皮伞 & Mycena latifolia (Peck) A. H. Sm. & 广叶小菇 \\
\hline Marasmiellus vaillantii (Pers.) Singer & 瓦氏微皮伞 & Mycena lohwagii Singer & 洛氏小菇 \\
\hline Marasmius albopurpureus T. H. Li \& C. Q. Wang & 银紫小皮伞 & Mycena maculata P. Karst. & 斑点小菇 \\
\hline Marasmius alutaceus Berk. \& M. A. Curtis & 淡棕小皮伞 & Mycena meliigena (Berk. \& Cooke) Sacc. & 楝小菇 \\
\hline Marasmius asiaticus Mešić \& Tkalčec & 亚洲小皮伞 & Mycena mellea Lj. N. Vassiljeva & 蜜黄小菇 \\
\hline Marasmius caryotae (Berk.) Petch & 核小皮伞 & Mycena niveipes (Murrill) Murrill & 白柄小菇 \\
\hline Marasmius limosus Quél. & 泥小皮伞 & Mycena overholtsii A. H. Sm. \& Solheim & 奥氏小菇 \\
\hline Marasmius parvulus Berk. \& M. A. Curtis & 细小皮伞 & Mycena phlogina Maas Geest. \& E. Horak & 焰小菇 \\
\hline Marasmius plicatus Wakker & 摺扇小皮伞 & Mycena pruni Velen. & 普兰小菇 \\
\hline Marasmius sparsifolius Chun Y. Deng \& T. H. Li & 疏叶小皮伞 & Mycena pterigena (Fr.) P. Kumm. & 䒴秆生小菇 \\
\hline $\begin{array}{l}\text { Marasmius subviridiphyllus Chun Y. Deng, } \\
\text { Y. H. Yang \& T. H. Li }\end{array}$ & 亚草绿小皮伞 & Mycena septentrionalis Maas Geest. & 北方小菇 \\
\hline Marasmius wynneae Berk. \& Broome & 维恩小皮伞 & Mycena stellaris Har. Takah., Taneyama \& Hadano & 星状小菇 \\
\hline $\begin{array}{l}\text { Megasporia ellipsoidea (B. K. Cui \& P. Du) } \\
\text { B. K. Cui \& Hai J. Li }\end{array}$ & 粗圆巨孔菌 & Mycena stipata Maas Geest. \& Schwöbel & 密小菇 \\
\hline $\begin{array}{l}\text { Megasporia violacea (B. K. Cui \& P. Du) B. K. Cui, } \\
\text { Y. C. Dai \& Hai J. Li }\end{array}$ & 紫巨孔菌 & |Mycena strobilicola J. Favre \& Kühner & 球果小菇 \\
\hline $\begin{array}{l}\text { Melanogaster intermedius (Berk.) Zeller \& } \\
\text { C. W. Dodge }\end{array}$ & 间型黑腹菌 & Mycena tenerrima (Berk.) Quél. & 柔弱小菇 \\
\hline $\begin{array}{l}\text { Melanohalea laciniatula (Flagey ex H. Olivier) } \\
\text { O. Blanco, A. Crespo, Divakar, Essl., D. Hawksw. \& } \\
\text { Lumbsch }\end{array}$ & 条裂黑尔衣 & Mycena tintinnabulum (Paulet) Quél. & 铃铛小菇 \\
\hline Melanoleuca congregata Bertault ex Contu & 聚生铦囊蘑 & Mycena ulmicola A. H. Sm. & 榆小菇 \\
\hline Melanoleuca humilis (Pers.) Pat. & 矮铦囊蘑 & Mycena vexans Sacc. & 针芽小菇 \\
\hline Melanoleuca tabularis Konrad & 扁盖铦囊蘑 & Mycenella bryophila (Voglino) Singer & 藓生孢微菇 \\
\hline Melanophyllum eyrei (Massee) Singer & 艾尔暗褶伞 & Mycoacia angustata H. S. Yuan & 狭针菌 \\
\hline Meruliopsis bella (Berk. \& M. A. Curtis) Ginns & 美丽拟干朽菌 & Mycoacia fuscoatra (Fr.) Donk & 暗针菌 \\
\hline Merulius insignis Wakef. & 突干朽菌 & Mycoacia odontoidea (Sheng H. Wu) Spirin \& Zmitr. & 齿状针菌 \\
\hline $\begin{array}{l}\text { Metarhizium owariense (Kobayasi) Kepler, } \\
\text { S. A. Rehner \& Humber }\end{array}$ & 尾张绿僵菌 & Mycobilimbia sabuletorum (Schreb.) Hafellner & 沙地菌盘衣 \\
\hline Mitremyces formosanus Sawada & 台湾丽球包 & Mycoleptodonoides vassiljevae Nikol. & 娃氏类小齿菌 \\
\hline
\end{tabular}


附录2 (续) Appendix 2 (continued)

\begin{tabular}{|c|c|c|c|}
\hline 拉丁学名 & 汉语学名 & 拉丁学名 & 汉语学名 \\
\hline Naucoria bohemica Velen. & 波地脆伞 & Peniophora reidii Boidin \& Lanq. & 里德隔孢伏革菌 \\
\hline Naucoria escharioides (Fr.) P. Kumm. & 污白脆伞 & Peniophora scintillans G. Cunn. & 闪伢隔孢伏革菌 \\
\hline Naucoria spadicea D. A. Reid & 束褐脆伞 & Peniophora septentrionalis Laurila & 北方隔狍伏革菌 \\
\hline Naucoria subconspersa Kühner ex P. D. Orton & 亚簇生脆伞 & Peniophora spathulata Sang H. Lin \& Z. C. Chen & 匙形隔狍伏革菌 \\
\hline Neoaleurodiscus monilifer (Malençon) Sheng H. Wu & 念珠新盘革菌 & Peniophora taiwanensis Sheng H. Wu & 台湾隔狍伏革菌 \\
\hline $\begin{array}{l}\text { Neoboletus sinensis (T. H. Li \& M. Zang) Gelardi, } \\
\text { Simonini \& Vizzini }\end{array}$ & 中国新牛肝菌 & $\begin{array}{l}\text { Peniophorella baculorubrensis (Gilb. \& M. Blackw.) } \\
\text { K. H. Larss. }\end{array}$ & $\begin{array}{l}\text { 红杆形小隔孢伏 } \\
\text { 革菌 }\end{array}$ \\
\hline $\begin{array}{l}\text { Neofomitella fumosipora (Corner) Y. C. Dai, Hai J. Li \& } \\
\text { Vlasák }\end{array}$ & 烟色新小层孔菌 & $\begin{array}{l}\text { Peniophorella echinocystis (J. Erikss. \& Å. Strid) } \\
\text { K. H. Larss. }\end{array}$ & $\begin{array}{l}\text { 刺囊小隔孢伏 } \\
\text { 革菌 }\end{array}$ \\
\hline Nidularia deformis (Willd.) Fr. & 变形鸟巢菌 & |Peniophorella guttulifera (P. Karst.) K. H. Larss. & 油小隔孢伏革菌 \\
\hline Nidularia pulvinata (Schwein.) Fr. & 垫状鸟巢菌 & Peniophorella neopubera (Sheng H. Wu) K. H. Larss. & $\begin{array}{l}\text { 软毛小隔孢伏 } \\
\text { 革菌 }\end{array}$ \\
\hline Odontia fibrosa (Berk. \& M. A. Curtis) Kõljalg & 纤维裂齿菌 & $\begin{array}{l}\text { Peniophorella subpraetermissa (Sheng H. Wu) } \\
\text { K. H. Larss. }\end{array}$ & $\begin{array}{l}\text { 亚坚实小隔孢伏 } \\
\text { 革菌 }\end{array}$ \\
\hline $\begin{array}{l}\text { Odonticium canoluteum (Sheng H. Wu) Zmitr. } \\
\text { \& Spirin }\end{array}$ & 土黄裂齿革菌 & Peniophorella tsugae (Burt) K. H. Larss. & $\begin{array}{l}\text { 铁杉小隔狍伏 } \\
\text { 革菌 }\end{array}$ \\
\hline $\begin{array}{l}\text { Odonticium flabelliradiatum } \\
\text { (J. Erikss. \& Hjortstam) Zmitr. }\end{array}$ & 扇形裂齿革菌 & $\begin{array}{l}\text { Perenniporia cystidiata Y. C. Dai, W. N. Chou \& } \\
\text { Sheng H. Wu }\end{array}$ & 囊多年卧孔菌 \\
\hline Omphalotus guepiniformis (Berk.) Neda & 棕褶类脐菇 & Perenniporia hainaniana B. K. Cui \& C. L. Zhao & 海南多年卧孔菌 \\
\hline $\begin{array}{l}\text { Ophiocordyceps corallomyces (Möller) G. H. Sung, } \\
\text { J. M. Sung, Hywel-Jones \& Spatafora }\end{array}$ & 珊瑚线虫草 & |Perenniporia lacerata B. K. Cui \& C. L. Zhao & 裂多年卧孔菌 \\
\hline $\begin{array}{l}\text { Ophiocordyceps entomorrhiza (Dicks.) G. H. Sung, } \\
\text { J. M. Sung, Hywel-Jones \& Spatafora }\end{array}$ & 虫根线虫草 & |Perenniporia luteola B. K. Cui \& C. L. Zhao & 浅黄多年卧孔菌 \\
\hline $\begin{array}{l}\text { Ophiocordyceps forquignonii (Quél.) G. H. Sung, } \\
\text { J. M. Sung, Hywel-Jones \& Spatafora }\end{array}$ & 福基尼翁线虫草 & |Perenniporia macropora B. K. Cui \& C. L. Zhao & 大孔多年卧孔菌 \\
\hline $\begin{array}{l}\text { Ophiocordyceps petchii (Mains) G. H. Sung, } \\
\text { J. M. Sung, Hywel-Jones \& Spatafora }\end{array}$ & 佩奇线虫草 & |Perenniporia phloiophila Gilb. \& M. Blackw. & 树皮多年卧孔菌 \\
\hline Oudemansiella crassifolia Corner & 厚小奥德蘑 & Perenniporia subtephropora B. K. Cui \& C. L. Zhao & $\begin{array}{l}\text { 亚白灰孢多年卧 } \\
\text { 孔菌 }\end{array}$ \\
\hline $\begin{array}{l}\text { Oudemansiella globospora (R. H. Petersen \& Nagas.) } \\
\text { Zhu L. Yang, G. M. Muell., G. Kost \& Rexer }\end{array}$ & 球狍小奥德蘑 & Perenniporia tianmuensis B. K. Cui \& C. L. Zhao & 天目多年卧孔菌 \\
\hline Pachylepyrium carbonicola (A. H. Sm.) Singer & 炭生厚壁狍伞 & Pezicula ericae (Sigler) P. R. Johnst. & 欧石楠无柄盘菌 \\
\hline Panellus orientalis (Kobayasi) Corner & 东方扇菇 & Pezicula magnispora Z. H. Zhong \& Zheng Wang & 巨狍无柄盘菌 \\
\hline Paxillus filamentosus (Scop.) Fr. & 丝桩菇 & Pezicula rubi (Lib.) Niessl & 鲁布无柄盘菌 \\
\hline Peltigera sibirica Gyeln. & 西伯利亚地卷 & Pezicula sporulosa Verkley & 多狍无柄盘菌 \\
\hline Peltigera subincusa (Gyeln.) Inumaru & 亚霜地卷 & |Peziza aurata (Le Gal) Spooner \& Y. J. Yao & 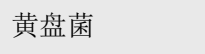 \\
\hline Peniophora alba Burt & 白隔狍伏革菌 & |Peziza badiofusca (Boud.) Dennis & 暗褐盘菌 \\
\hline Peniophora albobadia (Schwein.) Boidin & 白褐隔孢伏革菌 & Peziza cinatica Pfister & 糠状盘菌 \\
\hline Peniophora aurantiaca (Bres.) Höhn. \& Litsch. & 橙黄隔孢伏革菌 & Peziza gerardii Cooke & 杰勒德盘菌 \\
\hline Peniophora bicornis Hjortstam \& Ryvarden & 双角隔孢伏革菌 & Peziza hongkongensis Berk. \& M. A. Curtis & 香港盘菌 \\
\hline Peniophora borbonica Boidin \& Gilles & $\begin{array}{l}\text { 波尔本隔孢伏 } \\
\text { 革菌 }\end{array}$ & |Peziza limnaea Maas Geest. & 青萍盘菌 \\
\hline Peniophora ericina Bourdot & 直立隔孢伏革菌 & |Peziza natrophila A. Z. M. Khan & 喜碱盘菌 \\
\hline Peniophora incarnata (Pers.) P. Karst. & 肉色隔孢伏革菌 & Peziza ostracoderma Korf & 壳状盘菌 \\
\hline Peniophora malaiensis Boidin, Lanq. \& Gilles & 马来隔孢伏革菌 & |Peziza psammobia Rifai & 沙生盘菌 \\
\hline Peniophora manshurica Parmasto & 满洲隔孢伏革菌 & |Peziza queletii Medardi, Lantieri \& Cacialli & 凯莱盘菌 \\
\hline Peniophora ovalispora Boidin, Lanq. \& Gilles & 卵狍隔孢伏革菌 & Peziza rufescens Saut. & 暗红盘菌 \\
\hline Peniophora pseudonuda Hallenb. & 假裸隔狍伏革菌 & |Peziza verrucosa Pers. & 多疮盘菌 \\
\hline
\end{tabular}


附录2 (续) Appendix 2 (continued)

\begin{tabular}{|c|c|c|c|}
\hline 拉丁学名 & 汉语学名 & 拉丁学名 & 汉语学名 \\
\hline $\begin{array}{l}\text { Phaeoclavulina decolor (Berk. \& M. A. Curtis) } \\
\text { Giachini }\end{array}$ & 脱色暗锁瑚菌 & Phellinus betulinus (Murrill) Parmasto & 桦木层孔菌 \\
\hline $\begin{array}{l}\text { Phaeoclavulina mutabilis (Schild \& R. H. Petersen) } \\
\text { Giachini }\end{array}$ & 多变暗锁瑚菌 & |Phellinus caryophylleus (Cooke) Ryvarden & 石竹木层孔菌 \\
\hline $\begin{array}{l}\text { Phaeodaedalea incerta (Curr.) Țura, Zmitr., Wasser \& } \\
\text { Spirin }\end{array}$ & 未定暗迷孔菌 & Phellinus cyclobalanopsidis T. T. Chang \& W. N. Chou & 山槠木层孔菌 \\
\hline Phaeomarasmius erinaceus (Fr.) Scherff. ex Romagn. & 尖刺暗小皮伞 & |Phellinus deuteroprunicola T. T. Chang \& W. N. Chou & 李形木层孔菌 \\
\hline Phaeomarasmius rimulincola (Rabenh.) Scherff. & 裂纹暗小皮伞 & Phellinus formosanus T. T. Chang \& W. N. Chou & 台湾木层孔菌 \\
\hline Phaeophyscia confusa Moberg & 混黑蜈蚣衣 & |Phellinus neoquercinus M. J. Larsen & 新栋木层孔菌 \\
\hline Phaeophyscia denigrata (Hue) Moberg & 变黑蜈蚣衣 & |Phellinus pseudolaevigatus Parmasto ined. & 假光滑木层孔菌 \\
\hline $\begin{array}{l}\text { Phaeophyscia pyrrhophora (Poelt) D. D. Awasthi \& } \\
\text { M. Joshi }\end{array}$ & 火红黑蜈蚣衣 & Phellinus shoushanus T. T. Chang \& W. N. Chou & 寿山木层孔菌 \\
\hline Phaeotellus roseolus (Quél.) E. Horak & 玫红褐蘑 & Phellinus sonorae Gilb. & 亮金木层孔菌 \\
\hline $\begin{array}{l}\text { Phaeotremella neofoliacea (Chee J. Chen) Millanes \& } \\
\text { Wedin }\end{array}$ & 新茶暗银耳 & \begin{tabular}{|l} 
Phellinus sublaevigatus (Cleland \& Rodway) \\
P. K. Buchanan \& Ryvarden
\end{tabular} & 亚光滑木层孔菌 \\
\hline Phallus maderensis Calonge & 马德拉鬼笔 & Phellodon fuligineoalbus (J. C. Schmidt) R. E. Baird & 褐白栓齿菌 \\
\hline Phanerochaete aculeata Hallenb. & 刺原毛平革菌 & Phellorinia herculeana (Pers.) Kreisel & 赫氏歧裂灰包 \\
\hline Phanerochaete alba Sang H. Lin \& Z. C. Chen & 白原毛平革菌 & Phlebia coccineofulva Schwein. & 红桂射脉菌 \\
\hline Phanerochaete albida Sheng H. Wu & 微白原毛平革菌 & |Phlebia dictyophoroides Sang H. Lin \& Z. C. Chen & 网柄射脉菌 \\
\hline Phanerochaete angustocystidiata Sheng $\mathrm{H}$. Wu & 狭囊原毛平革菌 & |Phlebia pellucida Hjortstam \& Ryvarden & 透明射脉菌 \\
\hline Phanerochaete argillacea Sheng $\mathrm{H}$. Wu & 土色原毛平革菌 & \begin{tabular}{|l} 
Phlebia tuberculata (Berk. \& M. A. Curtis) Țura, \\
Zmitr., Wasser \& Spirin
\end{tabular} & 瘤射脉菌 \\
\hline Phanerochaete brunnea Sheng H. Wu & 褐原毛平革菌 & \begin{tabular}{|l} 
Phlebiopsis brunneocystidiata (Sheng H. Wu) \\
Miettinen
\end{tabular} & 褐囊拟射脉菌 \\
\hline Phanerochaete commixtoides Sang H. Lin \& Z. C. Chen & 混合原毛平革菌 & |Phlebiopsis himalayensis Dhingra & $\begin{array}{l}\text { 喜马拉雅拟射 } \\
\text { 脉菌 }\end{array}$ \\
\hline Phanerochaete eburnea Sheng H. Wu & $\begin{array}{l}\text { 象牙白原毛平 } \\
\text { 革菌 }\end{array}$ & $\begin{array}{l}\text { Phlebiopsis lamprocystidiata (Sheng H. Wu) } \\
\text { Sheng H. Wu \& Hallenb. }\end{array}$ & 光囊拟射脉菌 \\
\hline Phanerochaete flavidogrisea Sheng $\mathrm{H}$. Wu & 黄原毛平革菌 & |Phlebiopsis laxa (Sheng H. Wu) Miettinen & 松拟射脉菌 \\
\hline Phanerochaete fulva Sheng $\mathrm{H}$. Wu & 红黄原毛平革菌 & Phlebiopsis pilatii (Parmasto) Spirin \& Miettinen & 皮拉特拟射脉菌 \\
\hline Phanerochaete globosa Sang H. Lin \& Z. C. Chen & 球原毛平革菌 & |Pholiota conissans (Fr.) Kuyper \& Tjall.-Beuk. & 硫黄鳞伞 \\
\hline Phanerochaete hyphocystidiata Sheng H. Wu & 丝囊原毛平革菌 & Pholiota cubensis Earle & 古巴鳞伞 \\
\hline Phanerochaete inflata (B. S. Jia \& B. K. Cui) Miettinen & 肿原毛平革菌 & ||Pholiota jahnii Tjall.-Beuk. \& Bas & 扬氏鳞伞 \\
\hline Phanerochaete intertexta Sheng $\mathrm{H}$. Wu & 间型原毛平革菌 & |Pholiota parva A. Pearson & 细鳞伞 \\
\hline Phanerochaete odontoidea Sheng H. Wu & 齿状原毛平革菌 & Pholiota pedicellatum (Peck.) A. H. Smith \& Hesler & 小梗鳞伞 \\
\hline Phanerochaete parmastoi Sheng H. Wu & 帕氏原毛平革菌 & |Pholiota squarrosipes Cleland & 糠秕鳞伞 \\
\hline Phanerochaete reflexa Sheng $\mathrm{H}$. Wu & 反卷原毛平革菌 & |Phylloporia oblongospora Y. C. Dai \& H. S. Yuan & 长孢叶状层菌 \\
\hline Phanerochaete suballantoidea Sheng $\mathrm{H}$. Wu & $\begin{array}{l}\text { 亚腊肠原毛平 } \\
\text { 革菌 }\end{array}$ & Phylloporia spathulata (Hook.) Ryvarden & 匙形叶状层菌 \\
\hline Phanerochaete subglobosa Sheng H. Wu & $\begin{array}{l}\text { 亚球孢原毛平 } \\
\text { 革菌 }\end{array}$ & Phylloporus rhodophaeus Heinem. \& Rammeloo & 玫红褶孔菌 \\
\hline $\begin{array}{l}\text { Phaneroites subquercinus (Henn.) Hjortstam \& } \\
\text { Ryvarden }\end{array}$ & 亚栋原刺革菌 & Physalacria maipoensis Inderb. \& Desjardin & 迈坡泡头菌 \\
\hline $\begin{array}{l}\text { Phellinidium orientale (Bondartseva \& S. Herrera) } \\
\text { Bondartseva \& S. Herrera }\end{array}$ & 东方小木层孔菌 & Physcia convexella Moberg & 凸蜈蚣衣 \\
\hline Phellinopsis helwingiae L. W. Zhou \& W. M. Qin & $\begin{array}{l}\text { 青荚叶拟木层 } \\
\text { 孔菌 }\end{array}$ & Platygramme lueckingii Z. F. Jia \& Kalb & 吕金凸唇衣 \\
\hline
\end{tabular}


附录2 (续) Appendix 2 (continued)

\begin{tabular}{|c|c|c|c|}
\hline 拉丁学名 & 汉语学名 & |拉丁学名 & 汉语学名 \\
\hline Pleuroflammula chocoruensis Singer & 卓克拉侧火菇 & Psathyrella sphagnicola (Maire) J. Favre & 泥炭藓小脆柄菇 \\
\hline Pleurotus badius (Murrill) Murrill & 栗褐侧耳 & |Psathyrella spintrigera (Fr.) Konrad \& Maubl. & 连接小脆柄菇 \\
\hline Pleurotus subareolatus Peck & 网隙侧耳 & Psathyrella trepida (Fr.) Gillet & 胆小小脆柄菇 \\
\hline $\begin{array}{l}\text { Pleurotus tuoliensis (C. J. Mou) M. R. Zhao \& } \\
\text { Jin X. Zhang }\end{array}$ & 托里侧耳 & Pseudoboletus astraeicola (Imazeki) Šutara & 星假牛肝菌 \\
\hline Pluteus cinereofuscus J. E. Lange & 棕灰光柄菇 & |Pseudoboletus parasiticus (Bull.) Šutara & 寄生假牛肝菌 \\
\hline Pluteus exiguus (Pat.) Sacc. & 短小光柄菇 & $\begin{array}{l}\text { Pseudopiptoporus chocolatus (Lloyd) Decock \& } \\
\text { Ryvarden }\end{array}$ & 巧克力假滴孔菌 \\
\hline Pluteus hiatulus Romagn. & 裂光柄菇 & Pseudotomentella atrofusca M. J. Larsen & 黑褐假小垫革菌 \\
\hline Pluteus luctuosus Boud. & 卷缘光柄菇 & Pseudotomentella flavovirens (Höhn. \& Litsch.) Svrček & 黄绿假小垫革菌 \\
\hline Pluteus luteus (Redhead \& B. Liu) Redhead & 土黄光柄菇 & Pseudotomentella tristis (P. Karst.) M. J. Larsen & 暗假小垫革菌 \\
\hline Pluteus purpureofuscus Jiang Xu, T. H. Li \& Z. W. Ge & 紫褐光柄菇 & \begin{tabular}{|l} 
Pseudotremella nivalis (Chee J. Chen) X. Z. Liu, \\
F. Y. Bai, M. Groenew. \& Boekhout
\end{tabular} & 雪白假银耳 \\
\hline Pluteus romellii (Britzelm.) Sacc. & 罗梅尔光柄菇 & Psilocybe cinnamomea Yang K. Li, Y. Ye \& J. F. Liang & g肉桂色裸盖菇 \\
\hline Pluteus roseocandidus G. F. Atk. & 粉白光柄菇 & Psilocybe paupera Singer & 贫穷裸盖菇 \\
\hline Podoscypha brasiliensis D. A. Reid & 巴西柄杯菌 & Psilocybe subaeruginascens Höhn. & 亚铜绿裸盖菇 \\
\hline Podoscypha parvula (Lloyd) D. A. Reid & 小柄杯菌 & |Psilopezia nummularia Berk. & 硬币裸盘菌 \\
\hline Podoscypha thozetii (Berk.) Boidin & 托塞柄杯菌 & Pulveroboletus atkinsonianus (Murrill) L. D. Gómez & $\begin{array}{l}\text { 爱其逊粉末牛 } \\
\text { 肝菌 }\end{array}$ \\
\hline Podostroma giganteum S. Imai & 大肉座壳 & Radulodon subvinosus (Berk. \& Broome) Stalpers & 亚酒红齿舌革菌 \\
\hline Podostroma leucopus P. Karst. & 白肉座壳 & Ramalina pertusa Kashiw. & 穿孔树花 \\
\hline Polyporus cristulatus Speg. & 鸡冠多孔菌 & Ramalina requienii (De Not.) Jatta & 安魂树花 \\
\hline Polyporus formosus Laubert & 美丽多孔菌 & Ramaria americana (Corner) R. H. Petersen & 美洲枝瑚菌 \\
\hline Polyporus lipsiensis (Batsch) E. H. L. Krause & 利普西多孔菌 & |Ramaria brunneicontusa R. H. Petersen & 褐枝瑚菌 \\
\hline Polyporus subadmirabilis Bondartsev & 亚奇多孔菌 & Ramaria cystidiophora (Kauffman) Corner & 囊托枝瑚菌 \\
\hline Polyporus tuckahoe (Güssow) Lloyd & 获苓多孔菌 & Ramaria daucipes R. H. Petersen & 胡萝卜状枝瑚菌 \\
\hline Polystictus formosae Lloyd & 美丽云芝 & Ramaria flavicingula R. H. Petersen & 黄环枝瑚菌 \\
\hline Polystictus persoonii (Mont.) Cooke & 珀松云芝 & Ramaria flavosaponaria R. H. Petersen & 黄肥㿝草枝瑚菌 \\
\hline Porphyrellus atrofuscus E. A. Dick \& Snell & 黑褐红牛肝菌 & Ramaria foetida R. H. Petersen & 臭枝瑚菌 \\
\hline Porphyrellus formosanus K. W. Yeh \& Z. C. Chen & 台湾红牛肝菌 & |Ramaria incognita R. H. Petersen & 隐枝瑚菌 \\
\hline Porphyrellus fumosipes (Peck) Snell & 污柄红牛肝菌 & Ramaria lacteobrunnescens Schild & 乳褐枝瑚菌 \\
\hline $\begin{array}{l}\text { Porphyrellus holophaeus (Corner) Y. C. Li \& } \\
\text { Zhu L. Yang }\end{array}$ & 烟褐红牛肝菌 & Ramaria largentii Marr \& D. E. Stuntz & 拉根特枝瑚菌 \\
\hline Porphyrellus indecisus (Peck) E.-J. Gilbert & 橙变红牛肝菌 & Ramaria myceliosa (Peck) Corner & 菌丝状枝瑚菌 \\
\hline Postia brunnea Rajchenb. \& P. K. Buchanan & 褐泊氏孔菌 & Ramaria pallida (Schaeff.) Ricken & 苍白枝瑚菌 \\
\hline Postia subplacenta B. K. Cui & 饼形泊氏孔菌 & Ramaria pinicola (Burt) Corner & 松生枝瑚菌 \\
\hline $\begin{array}{l}\text { Protodontia piceicola (Kühner ex Bourdot) } \\
\text { G. W. Martin }\end{array}$ & 云杉原齿菌 & $\begin{array}{l}\text { Ramaria pulcherrima (Lj. N. Vassiljeva) } \\
\text { Lj. N. Vassiljeva ined. }\end{array}$ & 美极枝瑚菌 \\
\hline Psalliota sagata (Fr.) S. Petersen & 佐方腹草 & Ramaria purpurissima R. H. Petersen \& Scates & 紫枝瑚菌 \\
\hline Psathyrella fusca (J. E. Lange) A. Pearson & 暗小脆柄菇 & Ramaria rubella (Schaeff.) R. H. Petersen & 血红枝瑚菌 \\
\hline $\begin{array}{l}\text { Psathyrella gordonii (Berk. \& Broome) A. Pearson \& } \\
\text { Dennis }\end{array}$ & 戈登小脆柄菇 & Ramaria rubrievanescens Marr \& D. E. Stuntz & 红枝瑚菌 \\
\hline Psathyrella lutensis (Romagn.) Bon & 卢特小脆柄菇 & Ramaria sandaracina Marr \& D. E. Stuntz & 三陀罗枝瑚菌 \\
\hline Psathyrella pertinax (Fr.) Örstadius & 胶小脆柄菇 & Ramaria soluta (P. Karst.) Corner & 溶解枝瑚菌 \\
\hline Psathyrella pseudocasca (Romagn.) Kits van Wav. & 假皮小脆柄菇 & Ramaria testaceoflava (Bres.) Corner & 硬砖红枝瑚菌 \\
\hline Psathyrella scobinacea (Fr.) Konrad \& Maubl. & 锯屑小脆柄菇 & Ramariopsis asperulospora (G. F. Atk.) Corner & 散孢拟枝瑚菌 \\
\hline Psathyrella sphaerocystis P. D. Orton & 球囊小脆柄菇 & Ramariopsis pulchella (Boud.) Corner & 小丽拟枝瑚菌 \\
\hline
\end{tabular}


附录2 (续) Appendix 2 (continued)

\begin{tabular}{|c|c|c|c|}
\hline 拉丁学名 & 汉语学名 & 拉丁学名 & 汉语学名 \\
\hline Ramariopsis subtilis (Pers.) R. H. Petersen & 精细拟枝瑚菌 & $\begin{array}{l}\text { Saproamanita flavofloccosa (Nagas. \& Hongo) } \\
\text { Redhead, Vizzini, Drehmel \& Contu }\end{array}$ & 黄鳞腐生鹅膏 \\
\hline $\begin{array}{l}\text { Ramariopsis tenuicula (Bourdot \& Galzin) } \\
\text { R. H. Petersen }\end{array}$ & 纤细拟枝瑚菌 & $\begin{array}{l}\text { Saproamanita praegraveolens (Murrill) Redhead, } \\
\text { Vizzini, Drehmel \& Contu }\end{array}$ & 臭味腐生鹅膏 \\
\hline Ramariopsis tenuiramosa Corner & 细枝拟枝瑚菌 & Sarcodon fennicus (P. Karst.) P. Karst. & 芬氏肉齿菌 \\
\hline Rectipilus fasciculatus (Pers.) Agerer & 簇生似挂钟菌 & Sarcodon leucopus (Pers.) Maas Geest. \& Nannf. & 白肉齿菌 \\
\hline Repetobasidium intermedium Oberw. & 间型重担菌 & $\begin{array}{l}\text { Sarcodon martioflavus (Snell, K. A. Harrison \& } \\
\text { H. A. C. Jacks.) Maas Geest. }\end{array}$ & 黄肉齿菌 \\
\hline Resinomycena japonica Redhead \& Nagas. & 日本脂小菇 & Sarcodon thwaitesii (Berk. \& Broome) Maas Geest. & 大黄肉齿菌 \\
\hline Resupinatus striatulus (Pers.) Murrill & 条纹伏禇菌 & Sarcodon underwoodii Banker & 波状肉齿菌 \\
\hline Rhizomarasmius setosus (Sowerby) Antonín \& A. Urb. & 刚毛根皮伞 & Scleroderma australe Massee & 南方硬皮马勃 \\
\hline Rhodophana nitellina (Fr.) Papetti & 丽红柱菇 & Scleroderma poltaviense Sosin & 波地硬皮马勃 \\
\hline Rickenella swartzii (Fr.) Kuyper & 斯瓦氏藓菇 & Sclerotinia borealis Bubák \& Vleugel & 北方核盘菌 \\
\hline $\begin{array}{l}\text { Rigidoporus aureofulvus (Lloyd) P. K. Buchanan \& } \\
\text { Ryvarden }\end{array}$ & 黄褐硬孔菌 & Sclerotinia schinseng & 参核盘菌 \\
\hline Rigidoporus fibulatus H. S. Yuan \& Y. C. Dai & 扣状硬孔菌 & Scutellinia parvispora J. Moravec & 小孢盾盘菌 \\
\hline Rigidoporus undatus (Pers.) Donk & 波状硬孔菌 & Scutellinia subcervorum Svrček & 亚黄褐盾盘菌 \\
\hline Rimbachia arachnoidea (Peck) Redhead & 蛛丝缝伞 & Scutellinia superba (Velen.) Le Gal & 超盾盘菌 \\
\hline Rinodina roboris (Dufour ex Nyl.) Arnold & 栎饼干衣 & Scytinostroma lusitanicum (Trotter) P. M. Kirk & 卢西革垫菌 \\
\hline Roridomyces lamprosporus (Corner) Rexer & 光狍黏柄小菇 & Sebacina epigaea (Berk. \& Broome) Bourdot \& Galzin & 地生蜡壳菌 \\
\hline Rossbeevera yunnanensis Orihara \& M. E. Sm. & 云南罗叶腹菌 & Sidera lowei (Rajchenb.) Miettinen & 洛伊晶星革菌 \\
\hline Russula alnetorum Romagn. & 赤杨红菇 & $\begin{array}{l}\text { Sidera lunata (Romell ex Bourdot \& Galzin) } \\
\text { K. H. Larss. }\end{array}$ & 新月晶星革菌 \\
\hline Russula amoenolens Romagn. & 怡人红菇 & Simocybe haustellaris (Fr.) Watling & 霍氏绒盖伞 \\
\hline Russula brunneola Burl. & 褐红菇 & Sinoboletus magnisporus M. Zang \& C. M. Chen & 巨孢华牛肝菌 \\
\hline Russula changbaiensis G. J. Li \& H. A. Wen & 长白红菇 & Sistotrema subconfluens L. W. Zhou & 亚汇白齿菌 \\
\hline Russula chiui G. J. Li \& H. A. Wen & 㐮氏红菇 & Sistotremella perpusilla Hjortstam & 极小小白齿菌 \\
\hline Russula cuprea Krombh. & 铜色红菇 & Skeletocutis inflata B. K. Cui & 肿干皮孔菌 \\
\hline Russula jilinensis G. J. Li \& H. A. Wen & 吉林红菇 & Skeletocutis substellae Y. C. Dai & 亚星状干皮孔菌 \\
\hline Russula laeta Jul. Schäff. & 悦色红菇 & Skvortzovia furfurella (Bres.) Bononi \& Hjortstam & 鳞斯氏壳菌 \\
\hline Russula luteoviridans C. Martín & 黄绿红菇 & Sowerbyella imperialis (Peck) Korf & 壮丽索氏盘菌 \\
\hline Russula maculata Quél. & 斑点红菇 & $\begin{array}{l}\text { Steccherinum albofibrillosum (Hjortstam \& Ryvarden) } \\
\text { Hallenb. \& Hjortstam }\end{array}$ & 白线齿耳 \\
\hline Russula megaspora (Rodway) T. Lebel & 巨狍红菇 & Steccherinum cremicolor H. S. Yuan \& Sheng H. Wu & 奶油色齿耳 \\
\hline Russula odorata Romagn. & 香红菇 & Steccherinum elongatum H. S. Yuan \& Sheng H. Wu & 长齿耳 \\
\hline Russula olivina Ruots. \& Vauras & 橄榄绿红菇 & Stereum membranaceum Fr. & 膜㓞革菌 \\
\hline Russula pelargonia Niolle & 天答葵红菇 & Stereum ochraceoflavum (Schwein.) Sacc. & 黄褐㓞革菌 \\
\hline Russula persicina Krombh. & 桃红菇 & Stereum rugosiusculum Berk. \& M. A. Curtis & 皱纹㓞革菌 \\
\hline Russula polychroma Singer ex Hora & 杂色红菇 & Stropharia hardii G. F. Atk. & 哈德球盖菇 \\
\hline Russula subcompacta Britzelm. & 亚致密红菇 & Stropharia pseudocyanea (Desm.) Morgan & 假蓝球盖菇 \\
\hline Russula verna Singer & 春生红菇 & $\begin{array}{l}\text { Subulicystidium brachysporum (P. H. B. Talbot \& } \\
\text { V. C. Green) Jülich }\end{array}$ & 短狍雉囊菌 \\
\hline Russula vinosobrunnea (Bres.) Romagn. & 酒红褐红菇 & Subulicystidium meridense Oberw. & 梅里德雉囊菌 \\
\hline Russula zhejiangensis G. J. Li \& H. A. Wen & 浙江红菇 & Suillellus pictiformis Murrill & 着色小乳牛肝菌 \\
\hline Rutstroemia firma (Pers.) P. Karst. & 坚实蜡盘菌 & Suillellus rhodoxanthus (Krombh.) Blanco-Dios & 红黄小乳牛肝菌 \\
\hline Rutstroemia juglandis Raitv. & 脊蜡盘菌 & Suillus flavoluteus (Snell) Singer & 黄褐乳牛肝菌 \\
\hline $\begin{array}{l}\text { Rutstroemia petiolorum (Roberge ex Desm.) } \\
\text { W. L. White }\end{array}$ & 小柄蜡盘菌 & Suillus rubricontextus M. R. Ding \& H. A. Wen & 红肉乳牛肝菌 \\
\hline
\end{tabular}


附录2 (续) Appendix 2 (continued)

\begin{tabular}{|c|c|c|c|}
\hline 拉丁学名 & 汉语学名 & 拉丁学名 & 汉语学名 \\
\hline $\begin{array}{l}\text { Sutorius hainanensis (T. H. Li \& M. Zang) G. Wu \& } \\
\text { Zhu L. Yang }\end{array}$ & 海南紫盖牛肝菌 & Tremella laciniata With. & 条裂银耳 \\
\hline Sutorius junquilleus (Quél.) G. Wu \& Zhu L. Yang & 容氏紫盖牛肝菌 & |Tremella mesenterella Bandoni \& Ginns & 肠膜状银耳 \\
\hline $\begin{array}{l}\text { Sutorius obscureumbrinus (Hongo) G. Wu \& } \\
\text { Zhu L. Yang }\end{array}$ & 赫盖紫盖牛肝菌 & |Tremella occultifuroidea Chee J. Chen \& Oberw. & 隐叉银耳 \\
\hline $\begin{array}{l}\text { Sutorius tomentulosus (M. Zang, W. P. Liu \& M. R. Hu) } \\
\text { G. Wu \& Zhu L. Yang }\end{array}$ & ) 烟线紫盖牛肝菌 & Tremella resupinata Chee J. Chen & 扁平银耳 \\
\hline Sutorius venenatus (Nagas.) G. Wu \& Zhu L. Yang & 有毒紫盖牛肝菌 & ||Tremella sulcariae Diederich \& M. S. Christ. & 沟纹银耳 \\
\hline $\begin{array}{l}\text { Taiwanofungus salmoneus (T. T. Chang \& W. N. Chou) } \\
\text { Sheng H. Wu, Z. H. Yu, Y. C. Dai \& C. H. Su }\end{array}$ & 鲑色牛樟芝 & |Tremella taiwanensis Chee J. Chen & 台湾银耳 \\
\hline Tephrocybe mephitica (Fr.) M. M. Moser & 恶臭灰顶伞 & Tremella tropica Chee J. Chen & 热带银耳 \\
\hline Tephrocybe putida (P. Karst.) M. M. Moser & 腐败灰顶伞 & $\begin{array}{l}\text { Tretopileus sphaerophorus (Berk. \& M. A. Curtis) } \\
\text { S. Hughes \& Deighton }\end{array}$ & 球梗孔盖革菌 \\
\hline Tephrocybe striipilea (Fr.) Donk & 斯氏灰顶伞 & Trichaptum perrottetii (Lév.) Ryvarden & 佩氏附毛菌 \\
\hline Termitomyces congolensis (Beeli) Singer & 刚果蚁巢伞 & Tricholoma bambusarum Corner & 竹生口蘑 \\
\hline Termitomyces indicus Natarajan & 印度蚁巢伞 & Tricholoma striatum (Schaeff.) Quél. & 具纹口蘑 \\
\hline Termitomyces rutilans (Fr.) Sing. & 红橙蚁巢伞 & Tricholoma tenue P. W. Graff & 纤细口蘑 \\
\hline $\begin{array}{l}\text { Termitomyces sagittiformis (Kalchbr. \& Cooke) } \\
\text { D. A. Reid }\end{array}$ & 箭型蚁巢伞 & $\begin{array}{l}\text { Tricholosporum goniospermum (Bres.) Guzmán } \\
\text { ex T. J. Baroni }\end{array}$ & 棱孢十字孢口蘑 \\
\hline Termitomyces titanicus Pegler \& Piearce & 巨大蚁巢伞 & Tubaria pellucida (Bull.) Sacc. & 透明假脐菇 \\
\hline Termitomyces umkowaan (Cooke \& Massee) D. A. Reid & 乌姆科瓦蚁巢伞 & Tuber hubeiense L. Fan & 湖北块菌 \\
\hline Thelephora multifida Klotzsch & 多瓣裂革菌 & Tuber xanthomonosporum Qing \& Yun Wang bis & 黄狍块菌 \\
\hline Thelephora penicillata (Pers.) Fr. & 帚革菌 & Tubulicium vermiferum (Bourdot) Oberw. ex Jülich & 虫状管齿菌 \\
\hline Thelephora xerantha Berk. \& M. A. Curtis & 干革菌 & Tubulicrinis borealis J. Erikss. & 北方筒毛革菌 \\
\hline Tomentella bryophila (Pers.) M. J. Larsen & 蘚生小垫革菌 & Tubulicrinis confusus K. H. Larss. \& Hjortstam & 紊乱筒毛革菌 \\
\hline Tomentella coerulea Höhn. \& Litsch. & 蓝小垫革菌 & Tubulicrinis hamatus (H. S. Jacks.) Donk & 钩筒毛革菌 \\
\hline Tomentella ellisii (Sacc.) Jülich \& Stalpers & 埃尔默小垫革菌 & |Tubulicrinis sceptrifer (H. S. Jacks. \& Weresub) Donk & 权杖筒毛革菌 \\
\hline Tomentella ferruginea (Pers.) Pat. & 锈小垫革菌 & $\begin{array}{l}\text { Tubulicrinopsis farinacea (Boidin, Lanq. \& Gilles) } \\
\text { Kotir. \& Hjortstam }\end{array}$ & 粉拟筒毛革菌 \\
\hline Tomentella lateritia Pat. & 砖红小垫革菌 & Tulostoma obesum Cooke \& Ellis & 肉质柄灰包 \\
\hline Tomentella lilacinogrisea Wakef. & 灰紫小垫革菌 & Tulostoma simulans Lloyd & 相似柄灰包 \\
\hline Tomentella stuposa (Link) Stalpers & 卷毛小垫革菌 & Tylopilus atripurpureus (Corner) E. Horak & 暗紫粉孢牛肝菌 \\
\hline Tomentella terrestris (Berk. \& Broome) M. J. Larsen & 地生小垫革菌 & Tylopilus cervinicoccineus (Corner) E. Horak & 朱红粉孢牛肝菌 \\
\hline Tomentellopsis echinospora (Ellis) Hjortstam & 刺狍拟小垫革菌| & Tylopilus cutifractus (Corner) E. Horak & 皱盖粉孢牛肝菌 \\
\hline Torrubiella rostrata (Henn.) Sacc. \& D. Sacc. & 喙雉壳 & Tylopilus griseipurpureus (Corner) E. Horak & 灰紫粉孢牛肝菌 \\
\hline Trametes hirta (P. Beauv.) Zmitr., Wasser \& Ezhov & 多硬毛栓菌 & Tylopilus tristiculus (Massee) E. Horak & 线表粉孢牛肝菌 \\
\hline Trametes marianna (Pers.) Ryvarden & 玛丽安栓菌 & Typhula fistulosa (Holmsk.) Olariaga & 空管核瑚菌 \\
\hline Trametes mimetes (Wakef.) Ryvarden & 米梅栓菌 & Typhula phacorrhiza (Reichard) Fr. & 透根核瑚菌 \\
\hline Trametes neaniscus Berk. & 黄褐栓菌 & Typhula variabilis Riess & 多变核瑚菌 \\
\hline Trechispora cohaerens (Schwein.) Jülich \& Stalpers & 联柄糙狍孔菌 & Usnea diplotypa Vain. & 双型松萝 \\
\hline Trechispora dimitica Hallenb. & 二菌丝䊁孢孔菌 & Usnea flavocardia Räsänen & 黄褐松萝 \\
\hline Trechispora praefocata (Bourdot \& Galzin) Liberta & 球孢楉狍孔菌 & Vararia vassilievae Parmasto & 娃氏叉丝革菌 \\
\hline Trechispora rigida (Berk.) K. H. Larss. & 硬粘垉孔菌 & Veluticeps ambigua (Peck) Hjortstam \& Tellería & 含糊线柄革菌 \\
\hline Trechispora suberosa H. S. Yuan \& Y. C. Dai & 亚缺刻䊁孢孔菌 & |Verrucospora flavofusca (Henn.) Jülich & 黄褐疮狍伞 \\
\hline Tremella cerebriformis Chee J. Chen & 脑形银耳 & Volvariella surrecta (Knapp) Singer & 立起小包脚菇 \\
\hline Tremella flava Chee J. Chen & 金黄银耳 & Waitea circinata Warcup \& P. H. B. Talbot & 旋卷似串担革菌 \\
\hline Tremella fuscosuccinea Chee J. Chen & 琥珀银耳 & Wrightoporia nigrolimitata Jia J. Chen & 黑边赖特卧孔菌 \\
\hline
\end{tabular}


附录2 (续) Appendix 2 (continued)

\begin{tabular}{|c|c|c|c|}
\hline 拉丁学名 & 汉语学名 & 拉丁学名 & 汉语学名 \\
\hline Wrightoporia pouzarii A. David \& Rajchenb. & $\begin{array}{l}\text { 鲍扎尔赖特卧 } \\
\text { 孔菌 }\end{array}$ & Xylaria jiangsuensis G. Huang \& L. Guo & 江苏炭角菌 \\
\hline Wynnea sinensis B. Liu, M. H. Liu \& J. Z. Cao & 中国丛耳 & Xylaria juruensis Henn. & 茹鲁阿炭角菌 \\
\hline Xanthoconium separans (Peck) Halling \& Both & 裂管金狍牛肝菌 | & Xylaria leprosa Speg. & 粗粘炭角菌 \\
\hline Xanthoparmelia coreana (Gyeln.) Hale & 朝鲜黄梅 & Xylaria melanaxis Ces. & 黑轴炭角菌 \\
\hline Xenasma pruinosum (Pat.) Donk & 粉侧担菌 & $\begin{array}{l}\text { Xylaria moelleroclavus J. D. Rogers, Y. M. Ju \& } \\
\text { Hemmes }\end{array}$ & 棒炭角菌 \\
\hline Xenasma tulasnelloideum (Höhn. \& Litsch.) Donk & 涂氏侧担菌 & Xylaria multiplex (Kunze) Fr. & 笶炭角菌 \\
\hline Xenasmatella vaga (Fr.) Stalpers & 不定形小侧担菌|| & Xylaria myosurus Mont. & 松球炭角菌 \\
\hline $\begin{array}{l}\text { Xerocomellus intermedius (A. H. Sm. \& Thiers) } \\
\text { Svetash., Simonini \& Vizzini }\end{array}$ & $\begin{array}{l}\text { 间型小线盖牛 } \\
\text { 肝菌 }\end{array}$ & Xylaria ochraceostroma Y. M. Ju \& H. M. Hsieh & 赫子座炭角菌 \\
\hline Xerocomellus porosporus (Imler ex Watling) Šutara & $\begin{array}{l}\text { 孔孢小绒盖牛 } \\
\text { 肝菌 }\end{array}$ & Xylaria papulis Lloyd & 乳突炭角菌 \\
\hline Xerocomellus zelleri (Murrill) Klofac & $\begin{array}{l}\text { 泽勒小绒盖牛 } \\
\text { 肝菌 }\end{array}$ & Xylaria papyrifera (Link) Fr. & 纸质炭角菌 \\
\hline Xerocomus calocystides (Corner) E. Horak & $\begin{array}{l}\text { 美囊体线盖牛 } \\
\text { 肝菌 }\end{array}$ & \begin{tabular}{|l} 
Xylaria primorskensis Y. M. Ju, H. M. Hsieh, \\
Lar. N. Vassiljeva \& Akulov
\end{tabular} & 普氏炭角菌 \\
\hline Xerocomus junghuhnii (Höhn.) Singer & 容氏线盖牛肝菌|| & Xylaria regalis Cooke & 庄严炭角菌 \\
\hline Xerocomus satisfactus (Corner) E. Horak & 多褶绒盖牛肝菌|| & Xylaria schreuderiana Van der Byl & 斯克德勒炭角菌 \\
\hline Xerotus fuliginosus Berk. \& M. A. Curtis & 烟煤干菌 & Xylaria schwackei Henn. & 施魏克炭角菌 \\
\hline Xerotus pusillus Berk. \& M. A. Curtis & 小干菌 & Xylaria semiglobosa G. Huang \& L. Guo & 半圆炭角菌 \\
\hline Xylaria acuminatilongissima Y. M. Ju \& H. M. Hsieh & 长尖炭角菌 & Xylaria sphaerica G. Huang \& L. Guo & 球炭角菌 \\
\hline Xylaria alboareolata Y. M. Ju \& J. D. Rogers & 白纹炭角菌 & Xylaria striata Pat. & 具纹炭角菌 \\
\hline $\begin{array}{l}\text { Xylaria apoda (Berk. \& Broome) J. D. Rogers \& } \\
\text { Y. M. Ju }\end{array}$ & 无柄炭角菌 & Xylaria telfairii (Berk.) Sacc. & 戴尔菲炭角菌 \\
\hline Xylaria atrodivaricata Y. M. Ju \& H. M. Hsieh & 变黑炭角菌 & Xylaria wulaiensis Y. M. Ju \& Tzean & 乌兰炭角菌 \\
\hline Xylaria badia Pat. & 褐炭角菌 & $\begin{array}{l}\text { Xylodon anmashanensis (Yurchenko, H. X. Xiong \& } \\
\text { Sheng H. Wu) Riebesehl, Yurchenko \& Langer }\end{array}$ & 鞍马山趋木齿菌 \\
\hline Xylaria bambusicola Y. M. Ju \& J. D. Rogers & 竹生炭角菌 & $\begin{array}{l}\text { Xylodon apacheriensis (Gilb. \& Canf.) Hjortstam \& } \\
\text { Ryvarden }\end{array}$ & 阿帕奇趋木齿菌 \\
\hline Xylaria cirrata Pat. & 弯炭角菌 & \begin{tabular}{|l} 
Xylodon echinatus (Yurchenko \& Sheng H. Wu) \\
Riebesehl, Yurchenko \& Langer
\end{tabular} & 刺趋木齿菌 \\
\hline Xylaria dichotoma (Mont.) Mont. & 二叉炭角菌 & $\begin{array}{l}\text { Xylodon flaviporus (Berk. \& M. A. Curtis ex Cooke) } \\
\text { Riebesehl \& Langer }\end{array}$ & 浅黄趋木齿菌 \\
\hline Xylaria escharoidea (Berk.) Sacc. & 污白炭角菌 & $\begin{array}{l}\text { Xylodon hallenbergii (Sheng H. Wu) Hjortstam \& } \\
\text { Ryvarden }\end{array}$ & 哈氏趋木齿菌 \\
\hline Xylaria formosana Y. M. Ju \& Tzean & 台湾炭角菌 & $\begin{array}{l}\text { Xylodon heterocystidiatus (H. X. Xiong, Y. C. Dai \& } \\
\text { Sheng H. Wu) Riebesehl, Yurchenko \& Langer }\end{array}$ & 异囊趋木齿菌 \\
\hline Xylaria glebulosa (Ces.) Y. M. Ju \& J. D. Rogers & 产孢炭角菌 & \begin{tabular}{|l} 
Xylodon nudisetus (Warcup \& P. H. B. Talbot) \\
Hjortstam \& Ryvarden
\end{tabular} & 裸趋木齿菌 \\
\hline Xylaria griseosepiacea Y. M. Ju \& H. M. Hsieh & 灰黑炭角菌 & Xylodon ovisporus (Corner) Riebesehl \& Langer & 卵孢趋木齿菌 \\
\hline Xylaria hypoglossa Speg. & 光底炭角菌 & $\begin{array}{l}\text { Xylodon subclavatus (Yurchenko, H. X. Xiong \& } \\
\text { Sheng H. Wu) Riebesehl, Yurchenko \& Langer }\end{array}$ & 亚棒状趋木齿菌 \\
\hline $\begin{array}{l}\text { Xylaria intracolorata (J. D. Rogers, Callan \& Samuels) } \\
\text { J. D. Rogers \& Y. M. Ju }\end{array}$ & 色心炭角菌 & \multirow[t]{2}{*}{$\begin{array}{l}\text { Xylodon taiwanianus (Sheng H. Wu) Hjortstam \& } \\
\text { Ryvarden }\end{array}$} & \multirow[t]{2}{*}{ 台湾趋木齿菌 } \\
\hline Xylaria intraflava Y. M. Ju \& H. M. Hsieh & 黄肉炭角菌 & & \\
\hline
\end{tabular}


附录3《中国生物多样性红色名录一一型真菌卷》中菌物汉语学名修订

Appendix 3 Revised fungal Chinese names in Red List of China's Biodiversity-Macrofungi

\begin{tabular}{|c|c|c|c|}
\hline $\begin{array}{l}\text { 拉丁学名 } \\
\text { Scientific names }\end{array}$ & $\begin{array}{l}\text { 原汉语学名 } \\
\text { Original Chinese names }\end{array}$ & $\begin{array}{l}\text { 修订名 } \\
\text { Revised Chinese names }\end{array}$ & $\begin{array}{l}\text { 修订说明 } \\
\text { Revised notes }\end{array}$ \\
\hline Albatrellus citrinus & 桔黄地花菌 & 橘黄地花菌 & 误用字订正 \\
\hline Entoloma placidum & 温和粉褶萆 & 怡人粉褶蓸 & $\begin{array}{l}\text { 遵循优先律(2), 根据Rhodophyllus placidus怡人赤褶菇(中国科 } \\
\text { 学院微生物研究所, 1986)组合 }\end{array}$ \\
\hline Hemiphacidiaceae & 绿杯盘菌科 & 贫盘菌科 & $\begin{array}{l}\text { 遵循优先律(1), 来自《真菌名词及名称》(中国科学院微生物研 } \\
\text { 究所, 1986) }\end{array}$ \\
\hline Ithyphallus rugulosus & 皱白鬼笔 & 细皱白鬼笔 & $\begin{array}{l}\text { 遵循优先律(2), 根据Phallus rugulosus 细皱鬼笔(中国科学院微 } \\
\text { 生物研究所, 1986)组合 }\end{array}$ \\
\hline Lactarius aurantiacus & 桔色乳菇 & 橘色乳菇 & 误用字订正 \\
\hline Marasmius umboalbus & 脐状白小皮伞 & 白凸小皮伞 & 遵循优先律(3), 来自《中国大型菌物资源图鉴》(李玉等, 2015) \\
\hline Matula & 杯座菌属 & 杯座狍属 & $\begin{array}{l}\text { 遵循优先律(1), 来自《真菌名词及名称》(中国科学院微生物研 } \\
\text { 究所, 1986) }\end{array}$ \\
\hline Matula poroniiforme & 孔形杯座菌 & 孔形杯座孢 & $\begin{array}{l}\text { 遵循优先律(1), 来自《真菌名词及名称》(中国科学院微生物研 } \\
\text { 究所, 1986) }\end{array}$ \\
\hline Mycena citrinomarginata & 桔色凹小菇 & 橘色凹小菇 & 误用字订正 \\
\hline Phallus aurantiacus & 桔红鬼笔 & 橘红鬼笔 & 误用字订正 \\
\hline Phillipsia inaequalis & 桔色丕盘菌 & 橘色丕盘菌 & 误用字订正 \\
\hline Picipes badius & 黄褐黑斑根孔菌 & 褐黑斑根孔菌 & $\begin{array}{l}\text { 遵循优先律(4), 根据Polyporus badius褐多孔菌(张东柱等, 1989) } \\
\text { 修订 }\end{array}$ \\
\hline Pleurotus badius & 褐侧耳 & 栗褐侧耳 & $\begin{array}{l}\text { 原拟定汉语学名已被Pleurotus australis (孔祥辉等, 2008)使用, } \\
\text { 重新拟定 }\end{array}$ \\
\hline Sidera lenis & 晶星革菌 & 柔晶星革菌 & $\begin{array}{l}\text { 根据《真菌、地衣汉语学名命名法规》(中国菌物学会, 1987)第7 } \\
\text { 条修订 }\end{array}$ \\
\hline Steccherinaceae & 刺狍齿耳菌科 & 齿耳科 & $\begin{array}{l}\text { 根据《真菌、地衣汉语学名命名法规》(中国菌物学会, 1987)第5 } \\
\text { 条和Steccherinum齿耳属(中国科学院微生物研究所, 1986)修订 }\end{array}$ \\
\hline Stropholoma aurantiacum & 桔黄球盖菇 & 橘黄球盖菇 & 误用字订正 \\
\hline Termitomyces citriophyllus & 桔褶蚁巢伞 & 橘褶蚁巢伞 & 误用字订正 \\
\hline Tricholoma aurantium & 桔黄口蘑 & 橘黄口蘑 & 误用字订正 \\
\hline Ungulidaedalea fragilis & 蹄迷孔菌 & 脆蹄迷孔菌 & $\begin{array}{l}\text { 根据《真菌、地衣汉语学名命名法规》(中国菌物学会, 1987)第7 } \\
\text { 条修订 }\end{array}$ \\
\hline Urnula helvelloides & 脚瓶盘菌 & 马鞍状脚瓶盘菌 & $\begin{array}{l}\text { 根据《真菌、地衣汉语学名命名法规》(中国菌物学会, 1987)第7 } \\
\text { 条修订 }\end{array}$ \\
\hline Xanthoporia radiata & 辐射状金黄卧孔菌 & 辐射金黄卧孔菌 & $\begin{array}{l}\text { 根据《真菌、地衣汉语学名命名法规》(中国菌物学会, 1987)第 } \\
\text { 11条修订 }\end{array}$ \\
\hline
\end{tabular}

\section{参考文献}

Institute of Microbiology, Chinese Academy of Sciences (1986) A Glossary of Terms and Names of Fungi. Science Press, Beijing. (in Chinese) [中国科学院微生物研究所 (1986) 真菌名词及名称. 科学出版社, 北京.]

Kong XH, Zhang JC, Ma QF, Han ZH, Zhang PQ, Yang ZX (2008) Advances in the study of Ribonucleases proteins and peptides of mushrooms. Journal of Fungal Research, 6, 119-124. (in Chinese) [孔祥辉, 张介驰, 马庆芳, 韩增华, 张丕奇, 杨志兴 (2008) 草菌核糖核酸酶蛋白和肽的研究进展. 菌物研究, 6, 119-124.]

Li Y, Li TH, Yang ZL, Bau T, Dai YC (2015) Atlas of Chinese Macrofungal Resources. Central China Farmer's Publishing House, Zhengzhou. (in Chinese) [李玉, 李泰辉, 杨祝良, 图力古尔, 戴玉成 (2015) 中国大型菌物资源图鉴. 中原农民出版社, 郑州.]

Mycological Society of China (1987) Nomenclature Code of Fungal Chinese Names. Acta Mycologica Sinica, 6, 61-64. (in Chinese) [中国菌物学会 (1987) 真菌、地衣汉语学名命名法规. 真菌学报, 6, 61-64.]

Zhang DZ, Zhou WN, Wu ML, Wang YZ (1989) Macrofungi of Fushan. Agriculture Committee of the “Executivement”, Taipei. (in Chinese) [张东柱, 周文能, 吴美丽, 王也珍 (1989) 福山大型真菌. “行政院”农业委员会, 台北.] 